\title{
EXPANDING THE APPLICABILITY OF A TWO STEP NEWTON-TYPE PROJECTION METHOD FOR ILL-POSED PROBLEMS
}

\author{
Ioannis K. Argyros, Monnanda E. Shobha, Santhosh George
}

\begin{abstract}
There are many classes of ill-posed problems that cannot be solved with existing iterative methods, since the usual Lipschitz-type assumptions are not satisfied. In this study, we expand the applicability of a two step Newton-type projection method considered in [10], [11], using weaker assumptions. Numerical examples for the method and examples where the old assumptions are not satisfied but the new assumptions are satisfied are provided at the end of this study.
\end{abstract}

Keywords: Discretized Two Step Newton Tikhonov method, ill-posed Hammerstein-type operator equations, balancing principle, monotone operator, regularization method, projection method.

\section{Introduction}

This paper deals with the finite dimensional realization of a method considered in [10] for (nonlinear) Hammerstein-type equation

$$
K F(x)=f .
$$

Here $F: D(F) \subseteq X \rightarrow Z$ is nonlinear, $K: Z \rightarrow Y$ is a bounded linear operator $([7],[8])$ and $X, Z, Y$ are Hilbert spaces with inner product $\langle.,$.$\rangle and norm \|$. respectively.

We will assume that the problem (1.1) is ill-posed due to the non-closedness of the linear operator $K$ (see [9]). It is assumed that $f^{\delta} \in Y$ are the available noisy data with $\left\|f-f^{\delta}\right\| \leqslant \delta$ and $F$ possesses a uniformly bounded Fréchet derivative for each $x \in D(F)$, i.e.,

$$
\left\|F^{\prime}(x)\right\| \leqslant M, \quad x \in D(F)
$$

2010 Mathematics Subject Classification: primary: 47J06, 65J15, 47H17; secondary: 47A52, 65N20, 65J20 
for some $M$ (Here and below $F^{\prime}($.$) denotes the Fréchet derivative of F$ ). Observe that the solution $x$ of (1.1) with $f^{\delta}$ in place of $f$ can be obtained by first solving

$$
K z=f^{\delta}
$$

for $z$ and then solving the non-linear problem

$$
F(x)=z .
$$

In fact, in [10] we consider two cases of $F$, in the first case we assume that $F^{\prime}(x)^{-1}$ exist and in the second case we assume $F$ is monotone but $F^{\prime}(x)^{-1}$ does not exist. The method in [10] was a combination of Tikhonov regularization and Two Step Newton Method.

Regularization methods for ill-posed operator equation are usually defined in an infinite dimensional setting and have to be discretized for calculating a numerical solution [12]. Since finite dimensional problem are always well-posed in the sense of stable data dependence one could think of stabilizing an ill-posed problem by discretization. Regularization of ill-posed problems by projection methods can be found in literature for eg. in $[17,18,19]$. In this paper we consider the problem of approximately solving (1.1) in the finite dimensional setting of Hilbert spaces. Our goal is to expand the applicability of this method by weakening the usual assumptions for the convergence of these methods (see Assumption 3.1 and Assumption 3.2).

Recall [20], [21], that an operator $F$ is said to be monontone operator if $\langle F(x)-F(y), x-y\rangle \geqslant 0$ for all $x, y \in D(F)$.

The organization of this paper is as follows. Section 2 deals with Discretized Tikhonov regularization (detailed proof can be found in [11]) and Section 3 investigates the convergence of the Two Step Newton Tikhonov Projection Method (TSNTPM). Section 4 discusses the algorithm and finally the paper ends with a Numerical examples in Section 5.

\section{Discretized Tikhonov regularization}

This section deals with discretized Tikhonov regularized solution $z_{\alpha}^{h, \delta}$ of (1.2) and (an a priori and an a posteriori) error estimate for $\left\|F(\hat{x})-z_{\alpha}^{h, \delta}\right\|$.

The following assumption is used as in [8] to obtain the error estimate .

Assumption 2.1. There exists a continuous, strictly monotonically increasing function $\varphi:(0, a] \rightarrow(0, \infty)$ with $a \geqslant\|K\|^{2}$ satisfying;

- $\lim _{\lambda \rightarrow 0} \varphi(\lambda)=0$,

$$
\sup _{\lambda \geqslant 0} \frac{\alpha \varphi(\lambda)}{\lambda+\alpha} \leqslant \varphi(\alpha) \quad \forall \lambda \in(0, a]
$$

and

$$
F(\hat{x})-F\left(x_{0}\right)=\varphi\left(K^{*} K\right) w
$$

for some $w \in X$ such that $\|w\| \leqslant 1$. 
Let $\left\{P_{h}\right\}_{h>0}$ be a family of orthogonal projections on $X$. Let

$$
\begin{aligned}
\varepsilon_{h} & :=\left\|K\left(I-P_{h}\right)\right\|, \\
\tau_{h}: & =\left\|F^{\prime}(x)\left(I-P_{h}\right)\right\|, \quad \forall x \in D(F)
\end{aligned}
$$

and $\left\{b_{h}: h>0\right\}$ is such that $\lim _{h \rightarrow 0} \frac{\left\|\left(I-P_{h}\right) x_{0}\right\|}{b_{h}}=0, \lim _{h \rightarrow 0} \frac{\left\|\left(I-P_{h}\right) F\left(x_{0}\right)\right\|}{b_{h}}=0$ and $\lim _{h \rightarrow 0} b_{h}=0$. We assume that $\varepsilon_{h} \rightarrow 0$ and $\tau_{h} \rightarrow 0$ as $h \rightarrow 0$. The above assumption is satisfied if, $P_{h} \rightarrow I$ pointwise and if $K$ and $F^{\prime}(x)$ are compact operators. Further we assume that $\varepsilon_{h}<\varepsilon_{0}, \tau_{h} \leqslant \tau_{0}, b_{h} \leqslant b_{0}$ and $\delta \in\left(0, \delta_{0}\right]$.

The discretized Tikhonov regularization method for the regularized equation (1.2) consists of solving the equation

$$
\left(P_{h} K^{*} K P_{h}+\alpha P_{h}\right)\left(z_{\alpha_{k}}^{h, \delta}-P_{h} F\left(x_{0}\right)\right)=P_{h} K^{*}\left[f^{\delta}-K F\left(x_{0}\right)\right] .
$$

Theorem 2.2 (see [11], Theorem 2.4). Suppose Assumption 2.1 holds. Let $z_{\alpha_{k}}^{h, \delta}$ be as in (2.1) and $b_{h} \leqslant \frac{\delta+\varepsilon_{h}}{\sqrt{\alpha}}$. Then

$$
\left\|F(\hat{x})-z_{\alpha_{k}}^{h, \delta}\right\| \leqslant C\left(\varphi(\alpha)+\left(\frac{\delta+\varepsilon_{h}}{\sqrt{\alpha}}\right)\right)
$$

where $C=\frac{1}{2} \max \{M \rho, 1\}+1$.

\subsection{A priori choice of the parameter}

Note that the estimate $\varphi(\alpha)+\frac{\delta+\varepsilon_{h}}{\sqrt{ } \alpha}$ in $(2.2)$ is of optimal order for the choice $\alpha:=$ $\alpha(\delta, h)$ which satisfies $\varphi(\alpha(\delta, h))=\frac{\delta+\varepsilon_{h}}{\sqrt{\alpha(\delta, h)}}$. Let $\psi(\lambda):=\lambda \sqrt{\varphi^{-1}(\lambda)}, 0<\lambda \leqslant a$. Then we have $\delta+\varepsilon_{h}=\sqrt{\alpha(\delta, h)} \varphi(\alpha(\delta, h))=\psi(\varphi(\alpha(\delta, h)))$ and

$$
\alpha(\delta, h)=\varphi^{-1}\left(\psi^{-1}\left(\delta+\varepsilon_{h}\right)\right) .
$$

So the relation $(2.2)$ leads to $\left\|F(\hat{x})-z_{\alpha}^{h, \delta}\right\| \leqslant 2 C \psi^{-1}\left(\delta+\varepsilon_{h}\right)$.

\subsection{An adaptive choice of the parameter}

In this subsection, we consider the balancing principle established by Pereverzev and Shock [14] for choosing the parameter $\alpha$. Let

$$
D_{N}=\left\{\alpha_{i}: 0<\alpha_{0}<\alpha_{1}<\alpha_{2}<\cdots<\alpha_{N}\right\}
$$

be the set of possible values of the parameter $\alpha$.

Let

$$
\begin{aligned}
& l:=\max \left\{i: \varphi\left(\alpha_{i}\right) \leqslant \frac{\delta+\varepsilon_{h}}{\sqrt{\alpha_{i}}}\right\}<N, \\
& k=\max \left\{i: \alpha_{i} \in D_{N}^{+}\right\}
\end{aligned}
$$

where $D_{N}^{+}=\left\{\alpha_{i} \in D_{N}:\left\|z_{\alpha_{i}}^{\delta}-z_{\alpha_{j}}^{\delta}\right\| \leqslant \frac{4 C\left(\delta+\varepsilon_{h}\right)}{\sqrt{\alpha_{j}}}, j=0,1,2, \ldots, i-1\right\}$. 
We use the following theorem, the proof of which is analogous to the proof of Theorem 4.3 in [8], for our error analysis.

Theorem 2.3 (cf. [8, Theorem 4.3]). Let $l$ be as in (2.3), $k$ be as in (2.4) and $z_{\alpha_{k}}^{h, \delta}$ be as in (2.1) with $\alpha=\alpha_{k}$. Then $l \leqslant k$ and

$$
\left\|F(\hat{x})-z_{\alpha_{k}}^{h, \delta}\right\| \leqslant C\left(2+\frac{4 \mu}{\mu-1}\right) \mu \psi^{-1}\left(\delta+\varepsilon_{h}\right) .
$$

\section{Convergence analysis of the projection method}

In [11], [10], [8] the following Assumption was used, which is very difficult to verify (or does not hold) in general (see numerical examples at the last section of the paper)

Assumption 3.1 (cf.[16, Assumption 3 (A3)]). There exist a constant $k_{0} \geqslant 0$ such that for every $x, u \in D(F)$ and $v \in X$ there exists an element $\Phi(x, u, v) \in X$ such that $\left[F^{\prime}(x)-F^{\prime}(u)\right] v=F^{\prime}(u) \Phi(x, u, v),\|\Phi(x, u, v)\| \leqslant k_{0}\|v\|\|x-u\|$.

In the present paper we analyze the method by using a weaker Assumption than Assumption 3.1 and which is easier to verify:

Assumption 3.2. Let $x_{0} \in X$ be fixed. There exists a constant $K_{0} \geqslant 0$ such that for each $x, u \in D(F)$ and $v \in X$ there exists an element $\Phi(x, u, v) \in X$ depending on $x_{0}$ such that $\left[F^{\prime}(x)-F^{\prime}(u)\right] v=F^{\prime}(u) \Phi(x, u, v),\|\Phi(x, u, v)\| \leqslant K_{0}\|v\|(\| x-$ $\left.P_{h} x_{0}\|+\| u-P_{h} x_{0} \|\right)$.

Note that Assumption $3.1 \Rightarrow$ Assumption 3.2 but not necessarily vice versa.

\subsection{Case 1: TSNTPM when $F^{\prime}($.$) is invertible$}

In this section we assume that $F^{\prime}(x)$ is boundedly invertible for all $x \in D(F)$ i.e.,

$$
\left\|F^{\prime}(x)^{-1}\right\| \leqslant \beta_{1}
$$

for some $\beta_{1}>0$.

For an initial guess $x_{0} \in X$, the TSNTPM is defined iteratively as;

$$
\begin{aligned}
y_{n, \alpha_{k}}^{h, \delta} & =x_{n, \alpha_{k}}^{h, \delta}-P_{h} F^{\prime}\left(x_{n, \alpha_{k}}^{h, \delta}\right)^{-1} P_{h}\left(F\left(x_{n, \alpha_{k}}^{h, \delta}\right)-z_{\alpha_{k}}^{h, \delta}\right), \\
x_{n+1, \alpha_{k}}^{h, \delta} & =y_{n, \alpha_{k}}^{h, \delta}-P_{h} F^{\prime}\left(y_{n, \alpha_{k}}^{h, \delta}\right)^{-1} P_{h}\left(F\left(y_{n, \alpha_{k}}^{h, \delta}\right)-z_{\alpha_{k}}^{h, \delta}\right),
\end{aligned}
$$

where $x_{0, \alpha_{k}}^{h, \delta}:=P_{h} x_{0}$ and $z_{\alpha_{k}}^{h, \delta}$ is defined by (2.1) with $\alpha=\alpha_{k}$.

Note. Observe that if $b_{0}<\frac{1}{K_{0}}$ and if $x \in B_{r}\left(P_{h} x_{0}\right)$ where $r<\frac{1}{K_{0}}-b_{0}$, then $F^{\prime}(x)^{-1}$ exists and is bounded. This can be seen as follows:

$$
\begin{aligned}
\left\|F^{\prime}(x)^{-1}\right\| & =\sup _{\|v\| \leqslant 1}\left\|\left[I+F^{\prime}\left(x_{0}\right)^{-1}\left(F^{\prime}(x)-F^{\prime}\left(x_{0}\right)\right)\right]^{-1} F^{\prime}\left(x_{0}\right)^{-1} v\right\| \\
& \leqslant \sup _{\|v\| \leqslant 1} \frac{\left\|F^{\prime}\left(x_{0}\right)^{-1}\right\|}{1-\left\|F^{\prime}\left(x_{0}\right)^{-1}\left(F^{\prime}(x)-F^{\prime}\left(x_{0}\right)\right) v\right\|} .
\end{aligned}
$$


Now by Assumption 3.2 and the triangle inequality;

$$
\left\|x-x_{0}\right\| \leqslant\left\|x-P_{h} x_{0}\right\|+\left\|P_{h} x_{0}-x_{0}\right\|,
$$

we have

$$
\left\|F^{\prime}\left(x_{0}\right)^{-1}\left(F^{\prime}(x)-F^{\prime}\left(x_{0}\right)\right) v\right\| \leqslant K_{0}\left(r+b_{0}\right) .
$$

Hence by (3.1) and (3.4) we have

$$
\left\|F^{\prime}(x)^{-1}\right\| \leqslant \frac{\beta_{1}}{1-K_{0}\left(r+b_{0}\right)} .
$$

Thus without loss of generality we assume that

$$
\left\|F^{\prime}(x)^{-1}\right\| \leqslant \beta, \quad \forall x \in B_{r}\left(P_{h} x_{0}\right)
$$

and for some $\beta>0$.

Lemma 3.3. Let $x \in B_{r}\left(P_{h} x_{0}\right), b_{0}<\frac{1}{K_{0}}$ and $r<\frac{1}{K_{0}}-b_{0}$. Then we have $\left\|P_{h} F^{\prime}(x)^{-1} P_{h} F^{\prime}(x)\right\| \leqslant 1+\beta \tau_{0}$.

\section{Proof.}

$$
\begin{aligned}
\left\|P_{h} F^{\prime}(x)^{-1} P_{h} F^{\prime}(x)\right\|= & \sup _{\|v\| \leqslant 1}\left\|\left[P_{h} F^{\prime}(x)^{-1} P_{h} F^{\prime}(x)\right] v\right\| \\
\leqslant & \sup _{\|v\| \leqslant 1}\left\|P_{h} F^{\prime}(x)^{-1} P_{h} F^{\prime}(x)\left(P_{h}+I-P_{h}\right) v\right\| \\
\leqslant & \sup _{\|v\| \leqslant 1}\left\|\left[P_{h} F^{\prime}(x)^{-1} P_{h} F^{\prime}(x) P_{h}\right] v\right\| \\
& +\sup _{\|v\| \leqslant 1}\left\|P_{h} F^{\prime}(x)^{-1} P_{h} F^{\prime}(x) \times\left(I-P_{h}\right) v\right\| \\
\leqslant & 1+\beta \tau_{h} \leqslant 1+\beta \tau_{0} .
\end{aligned}
$$

Let

$$
e_{n, \alpha_{k}}^{h, \delta}:=\left\|y_{n, \alpha_{k}}^{h, \delta}-x_{n, \alpha_{k}}^{h, \delta}\right\|, \quad \forall n \geqslant 0 .
$$

Suppose that

$$
0<K_{0}<\frac{1}{4\left(1+\beta \tau_{0}\right)}
$$

and

$$
\frac{\delta_{0}+\varepsilon_{0}}{\sqrt{\alpha_{0}}}<\frac{2}{\beta(2 M+3)} .
$$

Let $\left\|\hat{x}-x_{0}\right\| \leqslant \rho$, with

$$
\rho<\frac{1}{M}\left(\frac{1}{\beta}-\left(\frac{3}{2}+M\right) \frac{\delta_{0}+\varepsilon_{0}}{\sqrt{\alpha_{0}}}\right)
$$

and

$$
\gamma_{\rho}:=\beta\left[M \rho+\left(\frac{3}{2}+M\right)\left(\frac{\varepsilon_{0}+\delta_{0}}{\sqrt{\alpha_{0}}}\right)\right],
$$


146 Ioannis K. Argyros, Monnanda E. Shobha, Santhosh George

and let $r$ be such that

$$
r \in\left(r_{1}, r_{2}\right)
$$

where

$$
r_{1}=\frac{1+\sqrt{1-16\left(1+\beta \tau_{0}\right) K_{0} \gamma_{\rho}}}{8\left(1+\beta \tau_{0}\right)}
$$

and

$$
\begin{aligned}
r_{2} & =\frac{1-\sqrt{1-16\left(1+\beta \tau_{0}\right) K_{0} \gamma_{\rho}}}{8\left(1+\beta \tau_{0}\right)} \\
b: & =4\left(1+\beta \tau_{0}\right) K_{0} r .
\end{aligned}
$$

Then, we have by (3.7)-(3.11) that

$$
0<\gamma_{\rho}<\frac{1}{16\left(1+\beta \tau_{0}\right) K_{0}}
$$

Theorem 3.4. Let $e_{n, \alpha_{k}}^{h, \delta}$ be as in equation (3.6) with $\delta \in\left(0, \delta_{0}\right], \alpha=\alpha_{k}$ and $\varepsilon_{h} \in\left(0, \varepsilon_{0}\right]$. Suppose the assumptions of Lemma 3.3 and Theorem 2.3 hold. Then, by Assumption 3.2, we have the following:

(a)

$$
\begin{aligned}
\left\|x_{n, \alpha_{k}}^{h, \delta}-y_{n-1, \alpha_{k}}^{h, \delta}\right\| \leqslant & \frac{K_{0}}{2}\left(1+\beta \tau_{0}\right)\left[3\left\|x_{n-1, \alpha_{k}}^{h, \delta}-x_{0, \alpha_{k}}^{h, \delta}\right\|\right. \\
& \left.+5\left\|y_{n-1, \alpha_{k}}^{h, \delta}-x_{0, \alpha_{k}}^{h, \delta}\right\|\right] e_{n-1, \alpha_{k}}^{h, \delta},
\end{aligned}
$$

(b)

$$
\begin{aligned}
\left\|x_{n, \alpha_{k}}^{h, \delta}-x_{n-1, \alpha_{k}}^{h, \delta}\right\| \leqslant & \left\{1+\frac{K_{0}}{2}\left(1+\beta \tau_{0}\right)\left[3\left\|x_{n-1, \alpha_{k}}^{h, \delta}-x_{0, \alpha_{k}}^{h, \delta}\right\|\right.\right. \\
& \left.\left.+5\left\|y_{n-1, \alpha_{k}}^{h, \delta}-x_{0, \alpha_{k}}^{h, \delta}\right\|\right]\right\} e_{n-1, \alpha_{k}}^{h, \delta},
\end{aligned}
$$

and

(c)

$$
\begin{aligned}
e_{n, \alpha_{k}}^{h, \delta} \leqslant & \frac{K_{0}}{2}\left(1+\beta \tau_{0}\right)\left[5\left\|x_{n, \alpha_{k}}^{h, \delta}-x_{0, \alpha_{k}}^{h, \delta}\right\|\right. \\
& \left.+3\left\|y_{n-1, \alpha_{k}}^{h, \delta}-x_{0, \alpha_{k}}^{h, \delta}\right\|\right]\left\|y_{n-1, \alpha_{k}}^{h, \delta}-x_{n, \alpha_{k}}^{h, \delta}\right\| \\
\leqslant & b^{2} e_{n-1, \alpha_{k}}^{h, \delta} \leqslant b^{2 n} e_{0, \alpha_{k}}^{h, \delta} \leqslant b^{2 n} \gamma_{\rho} .
\end{aligned}
$$


Proof. Observe that

$$
\begin{aligned}
& x_{n, \alpha_{k}}^{h, \delta}-y_{n-1, \alpha_{k}}^{h, \delta}= y_{n-1, \alpha_{k}}^{h, \delta}-x_{n-1, \alpha_{k}}^{h, \delta}-P_{h} F^{\prime}\left(y_{n-1, \alpha_{k}}^{h, \delta}\right)^{-1} P_{h}\left(F\left(y_{n-1, \alpha_{k}}^{h, \delta}\right)\right. \\
&\left.-z_{\alpha_{k}}^{h, \delta}\right)+P_{h} F^{\prime}\left(x_{n-1, \alpha_{k}}^{h, \delta}\right)^{-1} P_{h}\left(F\left(x_{n-1, \alpha_{k}}^{h, \delta}\right)-z_{\alpha_{k}}^{h, \delta}\right) \\
&= y_{n-1, \alpha_{k}}^{h, \delta}-x_{n-1, \alpha_{k}}^{h, \delta}-P_{h} F^{\prime}\left(y_{n-1, \alpha_{k}}^{h, \delta}\right)^{-1} P_{h}\left(F\left(y_{n-1, \alpha_{k}}^{h, \delta}\right)\right. \\
&\left.-F\left(x_{n-1, \alpha_{k}}^{h, \delta}\right)\right)-P_{h}\left[F^{\prime}\left(y_{n-1, \alpha_{k}}^{h, \delta}\right)^{-1}-F^{\prime}\left(x_{n-1, \alpha_{k}}^{h, \delta}\right)^{-1}\right] \\
& \times P_{h}\left(F\left(x_{n-1, \alpha_{k}}^{h, \delta}\right)-z_{\alpha_{k}}^{h, \delta}\right) \\
&= P_{h} F^{\prime}\left(y_{n-1, \alpha_{k}}^{h, \delta}\right)^{-1} P_{h} \\
& \times \int_{0}^{1}\left[F^{\prime}\left(y_{n-1, \alpha_{k}}^{h, \delta}\right)-F^{\prime}\left(x_{n-1, \alpha_{k}}^{h, \delta}+t\left(y_{n-1, \alpha_{k}}^{h, \delta}-x_{n-1, \alpha_{k}}^{h, \delta}\right)\right)\right] \\
& \times\left(y_{n-1, \alpha_{k}}^{h, \delta}-x_{n-1, \alpha_{k}}^{h, \delta}\right) d t+P_{h} F^{\prime}\left(y_{n-1, \alpha_{k}}^{h, \delta}\right)^{-1} \\
& \times P_{h}\left[F^{\prime}\left(x_{n-1, \alpha_{k}}^{h, \delta}\right)-F^{\prime}\left(y_{n-1, \alpha_{k}}^{h, \delta}\right)\right]\left(y_{n-1, \alpha_{k}}^{h, \delta}-x_{n-1, \alpha_{k}}^{h, \delta}\right) \\
&:= \Gamma_{1}+\Gamma_{2}, \\
& \text { where } P_{h} F^{\prime}\left(y_{n-1, \alpha_{k}}^{h, \delta}\right)^{-1} P_{h} \int_{0}^{1}\left[F^{\prime}\left(y_{n-1, \alpha_{k}}^{h, \delta}\right)-F^{\prime}\left(x_{n-1, \alpha_{k}}^{h, \delta}\right.\right. \\
&+\left.t\left(y_{n-1, \alpha_{k}}^{h, \delta}-x_{n-1, \alpha_{k}}^{h, \delta}\right)\right]\left(y_{n-1, \alpha_{k}}^{h, \delta}-x_{n-1, \alpha_{k}}^{h, \delta}\right) d t \\
& \Gamma_{1}
\end{aligned}
$$

and $\Gamma_{2}:=P_{h} F^{\prime}\left(y_{n-1, \alpha_{k}}^{h, \delta}\right)^{-1} P_{h}\left[F^{\prime}\left(x_{n-1, \alpha_{k}}^{h, \delta}\right)-F^{\prime}\left(y_{n-1, \alpha_{k}}^{h, \delta}\right)\right]\left(y_{n-1, \alpha_{k}}^{h, \delta}-x_{n-1, \alpha_{k}}^{h, \delta}\right)$. Note that by Assumption 3.2 and Lemma 3.3 we have

$$
\begin{aligned}
\left\|\Gamma_{1}\right\|= & \| P_{h} F^{\prime}\left(y_{n-1, \alpha_{k}}^{h, \delta}\right)^{-1} P_{h} \int_{0}^{1}\left[F^{\prime}\left(y_{n-1, \alpha_{k}}^{h, \delta}\right)-F^{\prime}\left(x_{n-1, \alpha_{k}}^{h, \delta}\right.\right. \\
& \left.+t\left(y_{n-1, \alpha_{k}}^{h, \delta}-x_{n-1, \alpha_{k}}^{h, \delta}\right)\right]\left(y_{n-1, \alpha_{k}}^{h, \delta}-x_{n-1, \alpha_{k}}^{h, \delta}\right) d t \| \\
\leqslant & \left(1+\beta \tau_{0}\right) \| \int_{0}^{1} \Phi\left(y_{n-1, \alpha_{k}}^{h, \delta}, x_{n-1, \alpha_{k}}^{h,,}+t\left(y_{n-1, \alpha_{k}}^{h, \delta}-x_{n-1, \alpha_{k}}^{h, \delta}\right),\right. \\
& \left.\times y_{n-1, \alpha_{k}}^{h, \delta}-x_{n-1, \alpha_{k}}^{h, \delta}\right) d t \| \\
\leqslant & K_{0}\left(1+\beta \tau_{0}\right)\left[\int_{0}^{1}\left\|x_{n-1, \alpha_{k}}^{h, \delta}-x_{0, \alpha_{k}}^{h, \delta}-t\left(y_{n-1, \alpha_{k}}^{h, \delta}-x_{n-1, \alpha_{k}}^{h, \delta}\right)\right\| d t\right. \\
& \left.+\left\|y_{n-1, \alpha_{k}}^{h, \delta}-x_{0, \alpha_{k}}^{h, \delta}\right\|\right]\left\|y_{n-1, \alpha_{k}}^{h, \delta}-x_{n-1, \alpha_{k}}^{h, \delta}\right\| \\
\leqslant & K_{0}\left(1+\beta \tau_{0}\right)\left[\int_{0}^{1}(1-t)\left\|x_{n-1, \alpha}^{h, \delta}-x_{0, \alpha_{k}}^{h, \delta}\right\|+t\left\|y_{n-1, \alpha_{k}}^{h, \delta}-x_{0, \alpha_{k}}^{h, \delta}\right\|\right. \\
& \left.+\left\|y_{n-1, \alpha_{k}}^{h, \delta}-x_{0, \alpha_{k}}^{h, \delta}\right\|\right] d t\left\|y_{n-1, \alpha_{k}}^{h, \delta}-x_{n-1, \alpha_{k}}^{h, \delta}\right\| \\
\leqslant & \frac{K_{0}}{2}\left(1+\beta \tau_{0}\right)\left[\left\|x_{n-1, \alpha_{k}}^{h, \delta}-x_{0, \alpha_{k}}^{h, \delta}\right\|+3\left\|y_{n-1, \alpha_{k}}^{h, \delta}-x_{0, \alpha_{k}}^{h, \delta}\right\|\right] e_{n-1, \alpha_{k}}^{h, \delta} .
\end{aligned}
$$


Similarly, we obtain

$$
\left\|\Gamma_{2}\right\| \leqslant K_{0}\left(1+\beta \tau_{0}\right)\left[\left\|y_{n-1, \alpha_{k}}^{h, \delta}-x_{0, \alpha_{k}}^{h, \delta}\right\|+\left\|x_{0, \alpha_{k}}^{h, \delta}-x_{n-1, \alpha_{k}}^{h, \delta}\right\|\right] e_{n-1, \alpha_{k}}^{h, \delta} .
$$

Hence from (3.15), (3.16) and (3.17), we get (a). Now (b) follows from (a) and the triangle inequality;

$$
\left\|x_{n, \alpha_{k}}^{h, \delta}-x_{n-1, \alpha_{k}}^{h, \delta}\right\| \leqslant\left\|x_{n, \alpha_{k}}^{h, \delta}-y_{n-1, \alpha_{k}}^{h, \delta}\right\|+\left\|y_{n-1, \alpha_{k}}^{h, \delta}-x_{n-1, \alpha_{k}}^{h, \delta}\right\| .
$$

To prove (c) we observe that

$$
\begin{aligned}
e_{n, \alpha_{k}}^{h, \delta}= & \| x_{n, \alpha_{k}}^{h, \delta}-y_{n-1, \alpha_{k}}^{h, \delta}-\left(P_{h} F^{\prime}\left(x_{n, \alpha_{k}}^{h, \delta}\right)\right)^{-1} P_{h}\left(F\left(x_{n, \alpha_{k}}^{h, \delta}\right)\right. \\
& \left.-z_{\alpha_{k}}^{h, \delta}\right)+P_{h} F^{\prime}\left(y_{n-1, \alpha_{k}}^{h, \delta}\right)^{-1} P_{h}\left(F\left(y_{n-1, \alpha_{k}}^{h, \delta}\right)-z_{\alpha_{k}}^{h, \delta}\right) \| \\
= & \| x_{n, \alpha_{k}}^{h, \delta}-y_{n-1, \alpha_{k}}^{h, \delta}-P_{h} F^{\prime}\left(x_{n, \alpha_{k}}^{h, \delta}\right)^{-1} P_{h}\left(F\left(x_{n, \alpha_{k}}^{h,,}\right)\right. \\
& \left.-F\left(y_{n-1, \alpha_{k}}^{h, \delta}\right)\right)+P_{h}\left[F^{\prime}\left(y_{n-1, \alpha_{k}}^{h, \delta}\right)^{-1}-F^{\prime}\left(x_{n, \alpha_{k}}^{h, \delta}\right)^{-1}\right] \\
& \times P_{h}\left(F\left(y_{n-1, \alpha_{k}}^{h, \delta}\right)-z_{\alpha_{k}}^{h, \delta}\right) \| \\
\leqslant & \Lambda_{1}+\Lambda_{2}
\end{aligned}
$$

where

$$
\Lambda_{1}:=\left\|x_{n, \alpha_{k}}^{h, \delta}-y_{n-1, \alpha_{k}}^{h, \delta}-P_{h} F^{\prime}\left(x_{n, \alpha_{k}}^{h, \delta}\right)^{-1} P_{h}\left(F\left(x_{n, \alpha_{k}}^{h, \delta}\right)-F\left(y_{n-1, \alpha_{k}}^{h, \delta}\right)\right)\right\|
$$

and

$$
\Lambda_{2}:=\left\|P_{h}\left[F^{\prime}\left(y_{n-1, \alpha_{k}}^{h, \delta}\right)^{-1}-F^{\prime}\left(x_{n, \alpha_{k}}^{h, \delta}\right)^{-1}\right] P_{h}\left(F\left(y_{n-1, \alpha_{k}}^{h, \delta}\right)-z_{\alpha_{k}}^{h, \delta}\right)\right\| .
$$

Analogous to the proof of (3.16) and (3.17), one can see that

$$
\Lambda_{1} \leqslant \frac{K_{0}}{2}\left(1+\beta \tau_{0}\right)\left[3\left\|x_{n, \alpha_{k}}^{h, \delta}-x_{0, \alpha_{k}}^{h, \delta}\right\|+\left\|y_{n-1, \alpha_{k}}^{h, \delta}-x_{0, \alpha_{k}}^{h, \delta}\right\|\right]\left\|x_{n, \alpha_{k}}^{h, \delta}-y_{n-1, \alpha_{k}}^{h, \delta}\right\|
$$

and

$$
\Lambda_{2} \leqslant K_{0}\left(1+\beta \tau_{0}\right)\left[\left\|x_{n, \alpha_{k}}^{h, \delta}-x_{0, \alpha_{k}}^{h, \delta}\right\|+\left\|y_{n-1, \alpha_{k}}^{h, \delta}-x_{0, \alpha_{k}}^{h, \delta}\right\|\right]\left\|x_{n, \alpha_{k}}^{h, \delta}-y_{n-1, \alpha_{k}}^{h, \delta}\right\| .
$$

Hence from (3.18) we obtain that

$$
\begin{aligned}
e_{n, \alpha_{k}}^{h, \delta} \leqslant & \frac{K_{0}}{2}\left(1+\beta \tau_{0}\right)\left[5\left\|x_{n, \alpha_{k}}^{h, \delta}-x_{0, \alpha_{k}}^{h, \delta}\right\|\right. \\
& \left.+3\left\|y_{n-1, \alpha_{k}}^{h, \delta}-x_{0, \alpha_{k}}^{h, \delta}\right\|\right]\left\|x_{n, \alpha_{k}}^{h, \delta}-y_{n-1, \alpha_{k}}^{h, \delta}\right\| \\
\leqslant & \frac{K_{0}}{2}\left(1+\beta \tau_{0}\right)(8 r) \frac{K_{0}}{2}\left(1+\beta \tau_{0}\right)(8 r)\left\|x_{n-1, \alpha_{k}}^{h, \delta}-y_{n-1, \alpha_{k}}^{h, \delta}\right\| \\
\leqslant & b^{2}\left\|x_{n-1, \alpha_{k}}^{h, \delta}-y_{n-1, \alpha_{k}}^{h, \delta}\right\| \\
\leqslant & b^{2 n} e_{0, \alpha_{k}}^{h, \delta} \leqslant b^{2 n} \gamma_{\rho} .
\end{aligned}
$$

This completes the proof of the Theorem. 
Theorem 3.5. Let $r$ be as defined in (3.10) and the assumptions of Theorem 3.4 hold. Then $x_{n, \alpha_{k}}^{h, \delta}, y_{n, \alpha_{k}}^{h, \delta} \in B_{r}\left(P_{h} x_{0}\right)$, for all $n \geqslant 0$.

Proof. Note that by (b) of Theorem 3.4 we have,

$$
\begin{aligned}
\left\|x_{1, \alpha_{k}}^{h, \delta}-P_{h} x_{0}\right\| & =\left\|x_{1, \alpha_{k}}^{h, \delta}-x_{0, \alpha_{k}}^{h, \delta}\right\| \\
& \leqslant\left[1+\left(1+\beta \tau_{0}\right) \frac{K_{0}}{2}(8 r)\right] \gamma_{\rho} \\
& \leqslant(1+b) \gamma_{\rho} \\
& \leqslant \frac{\gamma_{\rho}}{1-b}<r,
\end{aligned}
$$

i.e., $x_{1, \alpha_{k}}^{h, \delta} \in B_{r}\left(P_{h} x_{0}\right)$. Again note that from (3.20) and Theorem 3.4 we get,

$$
\begin{aligned}
\left\|y_{1, \alpha_{k}}^{h, \delta}-P_{h} x_{0}\right\| & \leqslant\left\|y_{1, \alpha_{k}}^{h, \delta}-x_{1, \alpha_{k}}^{h, \delta}\right\|+\left\|x_{1, \alpha_{k}}^{h, \delta}-P_{h} x_{0}\right\| \\
& \leqslant\left[1+\left(1+\beta \tau_{0}\right) 4 K_{0} r+\left(\left(1+\beta \tau_{0}\right) 4 K_{0} r\right)^{2}\right] \gamma_{\rho} \\
& \leqslant\left(1+b+b^{2}\right) \gamma_{\rho} \\
& \leqslant \frac{\gamma_{\rho}}{1-b}<r
\end{aligned}
$$

i.e., $y_{1, \alpha_{k}}^{h, \delta} \in B_{r}\left(P_{h} x_{0}\right)$. Further by (3.20) and (b) of Theorem 3.4 we have,

$$
\begin{aligned}
\left\|x_{2, \alpha_{k}}^{h, \delta}-P_{h} x_{0}\right\| & \leqslant\left\|x_{2, \alpha_{k}}^{h, \delta}-x_{1, \alpha_{k}}^{h, \delta}\right\|+\left\|x_{1, \alpha_{k}}^{h, \delta}-P_{h} x_{0}\right\| \\
& \leqslant(1+b) e_{1, \alpha_{k}}^{h, \delta}+(1+b) \gamma_{\rho} \\
& \leqslant\left(1+b+b^{2}+b^{3}\right) \gamma_{\rho} \\
& \leqslant \frac{1}{1-b} \gamma_{\rho}<r
\end{aligned}
$$

and

$$
\begin{aligned}
\left\|y_{2, \alpha_{k}}^{h, \delta}-P_{h} x_{0}\right\| & \leqslant\left\|y_{2, \alpha_{k}}^{h, \delta}-x_{2, \alpha_{k}}^{h, \delta}\right\|+\left\|x_{2, \alpha_{k}}^{h, \delta}-P_{h} x_{0}\right\| \\
& \leqslant b^{4} \gamma_{\rho}+\left(1+b+b^{2}+b^{3}\right) \gamma_{\rho} \\
& \leqslant\left(1+b+b^{2}+b^{3}+b^{4}\right) \gamma_{\rho} \\
& \leqslant \frac{1}{1-b} \gamma_{\rho}<r
\end{aligned}
$$

by the choice of $r$, i.e., $x_{2, \alpha_{k}}^{h, \delta}, y_{2, \alpha_{k}}^{h, \delta} \in B_{r}\left(P_{h} x_{0}\right)$. Continuing this way one can prove that $x_{n, \alpha_{k}}^{h, \delta}, y_{n, \alpha_{k}}^{h, \delta} \in B_{r}\left(P_{h} x_{0}\right), \forall n \geqslant 0$. This completes the proof.

Theorem 3.6. Let $y_{n, \alpha_{k}}^{h, \delta}$ and $x_{n, \alpha_{k}}^{h, \delta}$ be as in (3.2) and (3.3) respectively and hypotheses of Theorem 3.5 hold. Then $\left(x_{n, \alpha_{k}}^{h, \delta}\right)$ is a Cauchy sequence in $B_{r}\left(P_{h} x_{0}\right)$ and converges to $x_{\alpha_{k}}^{h, \delta} \in \overline{B_{r}\left(P_{h} x_{0}\right)}$. Further $P_{h} F\left(x_{\alpha_{k}}^{h, \delta}\right)=z_{\alpha_{k}}^{h, \delta}$ and

$$
\left\|x_{n, \alpha_{k}}^{h, \delta}-x_{\alpha_{k}}^{h, \delta}\right\| \leqslant \frac{(1+b) b^{2 n} \gamma_{\rho}}{1-b^{2}}
$$

where $\gamma_{\rho}$ and $b$ are defined by (3.9) and (3.11), respectively. 
Proof. Using the relation (b) and (c) of Theorem 3.4, we obtain

$$
\begin{aligned}
\left\|x_{n+i+1, \alpha_{k}}^{h, \delta}-x_{n+i, \alpha_{k}}^{h, \delta}\right\| & \leqslant(1+b) b^{0}\left\|x_{n+i, \alpha_{k}}^{h, \delta}-y_{n+i, \alpha_{k}}^{h, \delta}\right\| \\
& \leqslant(1+b) b\left\|x_{n+i, \alpha_{k}}^{h, \delta}-y_{n+i-1, \alpha_{k}}^{h, \delta}\right\| \\
& \leqslant(1+b) b^{2}\left\|x_{n+i-1, \alpha_{k}}^{h, \delta}-y_{n+i-1, \alpha_{k}}^{h, \delta}\right\| \\
& \leqslant(1+b) b^{2(n+i)} e_{0, \alpha_{k}}^{h, \delta} \\
& \leqslant(1+b) b^{2(n+i)} \gamma_{\rho} .
\end{aligned}
$$

So,

$$
\begin{aligned}
\left\|x_{n+m, \alpha_{k}}^{h, \delta}-x_{n, \alpha_{k}}^{h, \delta}\right\| & \leqslant \sum_{i=0}^{m-1}\left\|x_{n+i+1, \alpha_{k}}^{h, \delta}-x_{n+i, \alpha_{k}}^{h, \delta}\right\| \\
& \leqslant(1+b) b^{2 n} \sum_{i=0}^{m-1} b^{2 i} \\
& =(1+b) b^{2 n} \frac{1-b^{2 m}}{1-b^{2}} \gamma_{\rho} \rightarrow \frac{(1+b) b^{2 n}}{1-b^{2}} \gamma_{\rho}
\end{aligned}
$$

as $m \rightarrow \infty$. Observe that from $(3.2)$

$$
\begin{aligned}
\left\|P_{h}\left(F\left(x_{n, \alpha_{k}}^{h, \delta}\right)-z_{\alpha_{k}}^{h, \delta}\right)\right\| & =\left\|P_{h} F^{\prime}\left(x_{n, \alpha_{k}}^{h, \delta}\right)\left(x_{n, \alpha_{k}}^{h, \delta}-y_{n, \alpha_{k}}^{h, \delta}\right)\right\| \\
& \leqslant\left\|F^{\prime}\left(x_{n, \alpha_{k}}^{h, \delta}\right)\right\|\left\|y_{n, \alpha_{k}}^{h, \delta}-x_{n, \alpha_{k}}^{h, \delta}\right\| \\
& \leqslant M e_{n, \alpha_{k}}^{h, \delta}
\end{aligned}
$$

Now by letting $n \rightarrow \infty$ in (3.21) we obtain $P_{h} F\left(x_{\alpha_{k}}^{h, \delta}\right)=z_{\alpha_{k}}^{h, \delta}$. This completes the proof.

Hereafter we assume that

$$
\rho \leqslant r<\frac{1}{\left(1+\beta \tau_{0}\right) K_{0}}
$$

Theorem 3.7. Suppose $\left(1+\beta \tau_{0}\right) K_{0} r<1$ and Assumption 2.1 and 3.2 hold. Then

$$
\left\|\hat{x}-x_{\alpha_{k}}^{h, \delta}\right\| \leqslant \frac{\beta}{\left(1-\left(1+\beta \tau_{0}\right) K_{0} r\right)}\left\|F(\hat{x})-z_{\alpha_{k}}^{h, \delta}\right\|
$$


Proof. Observe that

$$
\begin{aligned}
\left\|\hat{x}-x_{\alpha_{k}}^{h, \delta}\right\|= & \left\|\hat{x}-x_{\alpha_{k}}^{h, \delta}+P_{h} F^{\prime}\left(P_{h} x_{0}\right)^{-1} P_{h}\left[F\left(x_{\alpha_{k}}^{h, \delta}\right)-F(\hat{x})+F(\hat{x})-z_{\alpha_{k}}^{h, \delta}\right]\right\| \\
\leqslant & \| P_{h} F^{\prime}\left(P_{h} x_{0}\right)^{-1}\left[P_{h} F^{\prime}\left(P_{h} x_{0}\right)\left(\hat{x}-x_{\alpha_{k}}^{h, \delta}\right)-P_{h}(F(\hat{x})\right. \\
& \left.-F\left(x_{\alpha_{k}}^{h, \delta}\right)\right]\|+\| P_{h} F^{\prime}\left(P_{h} x_{0}\right)^{-1} P_{h}\left(F(\hat{x})-z_{\alpha_{k}}^{h, \delta}\right) \| \\
\leqslant & \| P_{h} F^{\prime}\left(P_{h} x_{0}\right)^{-1} P_{h} \int_{0}^{1}\left[F^{\prime}\left(P_{h} x_{0}\right)-F^{\prime}\left(\hat{x}+t\left(x_{\alpha_{k}}^{h, \delta}-\hat{x}\right)\right)\right] \\
& \times\left(\hat{x}-x_{\alpha_{k}}^{h, \delta}\right) d t\|+\| P_{h} F^{\prime}\left(P_{h} x_{0}\right)^{-1} P_{h}\left(F(\hat{x})-z_{\alpha_{k}}^{h, \delta}\right) \| \\
\leqslant & \left\|P_{h} F^{\prime}\left(P_{h} x_{0}\right)^{-1} P_{h} F^{\prime}\left(P_{h} x_{0}\right)\right\| \int_{0}^{1} \Phi\left(P_{h} x_{0}, \hat{x}+t\left(x_{\alpha_{k}}^{h, \delta}-\hat{x}\right),\right. \\
& \left.\hat{x}-x_{\alpha_{k}}^{h, \delta}\right) d t\|+\| P_{h} F^{\prime}\left(P_{h} x_{0}\right)^{-1} P_{h}\left(F(\hat{x})-z_{\alpha_{k}}^{h, \delta}\right) \| \\
\leqslant & \left(1+\beta \tau_{0}\right) K_{0} r\left\|\hat{x}-x_{\alpha_{k}}^{h, \delta}\right\|+\beta\left\|F(\hat{x})-z_{\alpha_{k}}^{h, \delta}\right\| .
\end{aligned}
$$

The last step follows from Assumption 3.2, Lemma 3.3, (3.5) and the relation $\left\|P_{h} x_{0}-\hat{x}-t\left(x_{\alpha_{k}}^{h, \delta}-\hat{x}\right)\right\| \leqslant r$. This completes the proof.

The following Theorem is a consequence of Theorem 3.6 and Theorem 3.7.

Theorem 3.8. Let $x_{n, \alpha_{k}}^{h, \delta}$ be as in (3.3), assumptions in Theorem 3.6 and Theorem 3.7 hold. Then

$$
\left\|\hat{x}-x_{n, \alpha_{k}}^{h, \delta}\right\| \leqslant \frac{(1+b) b^{2 n} \gamma_{\rho}}{1-b^{2}}+\frac{\beta}{\left(1-\left(1+\beta \tau_{0}\right) K_{0} r\right)}\left\|F(\hat{x})-z_{\alpha_{k}}^{h, \delta}\right\|
$$

where $\gamma_{\rho}$ is as in Theorem 3.6.

Now since $l \leqslant k$ and $\alpha_{\delta} \leqslant \alpha_{l+1} \leqslant \mu \alpha_{l}$ we have

$$
\frac{\delta+\varepsilon_{h}}{\sqrt{\alpha_{k}}} \leqslant \frac{\delta+\varepsilon_{h}}{\sqrt{\alpha_{l}}} \leqslant \mu \frac{\delta+\varepsilon_{h}}{\sqrt{\alpha_{\delta}}}=\mu \varphi(\alpha(\delta, h))=\mu \psi^{-1}\left(\delta+\varepsilon_{h}\right) .
$$

This leads to the following theorem,

Theorem 3.9. Let $x_{n, \alpha_{k}}^{h, \delta}$ be as in (3.3), assumptions in Theorem 3.8 hold. Let

$$
n_{k}:=\min \left\{n: b^{2 n} \leqslant \frac{\delta+\varepsilon_{h}}{\sqrt{\alpha_{k}}}\right\} .
$$

Then

$$
\left\|\hat{x}-x_{n_{k}, \alpha_{k}}^{h, \delta}\right\|=O\left(\psi^{-1}\left(\delta+\varepsilon_{h}\right)\right)
$$

\subsection{Case 2: TSNTPM when $F$ is monotone and $F^{\prime}($.$) is non-invertible$}

Assumption 3.10. There exists a continuous, strictly monotonically increasing function $\varphi_{1}:(0, b] \rightarrow(0, \infty)$ with $b \geqslant\left\|F^{\prime}\left(x_{0}\right)\right\|$ satisfying;

- $\lim _{\lambda \rightarrow 0} \varphi_{1}(\lambda)=0$, 


$$
\sup _{\lambda \geqslant 0} \frac{\alpha \varphi_{1}(\lambda)}{\lambda+\alpha} \leqslant \varphi_{1}(\alpha) \quad \forall \lambda \in(0, b]
$$

and

- there exists $v \in X$ with $\|v\| \leqslant 1$ (cf.[13]) such that

$$
x_{0}-\hat{x}=\varphi_{1}\left(F^{\prime}\left(x_{0}\right)\right) v .
$$

- for each $x \in B_{r}\left(x_{0}\right):=\left\{x:\left\|x-x_{0}\right\|<r\right\}$ there exists a bounded linear operator $G\left(x, x_{0}\right)$ (cf.[15]) such that

$$
F^{\prime}(x)=F^{\prime}\left(x_{0}\right) G\left(x, x_{0}\right)
$$

with $\left\|G\left(x, x_{0}\right)\right\| \leqslant K_{1}$.

First we consider an iterative method to approximate the zero $x_{c, \alpha_{k}}^{h, \delta}$ of

$$
P_{h}\left(F(x)+\frac{\alpha_{k}}{c}\left(x-x_{0}\right)\right)=P_{h} z_{\alpha_{k}}^{h, \delta}
$$

and then we show that $x_{c, \alpha_{k}}^{h, \delta}$ is an approximation to the solution $\hat{x}$ of (1.1) where $c \leqslant \alpha_{k}$. For an initial guess $x_{0} \in X$ and for $R(x):=P_{h} F^{\prime}(x) P_{h}+\frac{\alpha_{k}}{c} P_{h}$, the iterative method is defined as:

$$
\tilde{y}_{n, \alpha_{k}}^{h, \delta}=\tilde{x}_{n, \alpha_{k}}^{h, \delta}-R\left(\tilde{x}_{n, \alpha_{k}}^{h, \delta}\right)^{-1} P_{h}\left[F\left(\tilde{x}_{n, \alpha_{k}}^{h, \delta}\right)-z_{\alpha_{k}}^{h, \delta}+\frac{\alpha_{k}}{c}\left(\tilde{x}_{n, \alpha_{k}}^{h, \delta}-\tilde{x}_{0, \alpha_{k}}^{h, \delta}\right)\right]
$$

and

$$
\tilde{x}_{n+1, \alpha_{k}}^{h, \delta}=\tilde{y}_{n, \alpha_{k}}^{h, \delta}-R\left(\tilde{y}_{n, \alpha_{k}}^{h, \delta}\right)^{-1} P_{h}\left[F\left(\tilde{y}_{n, \alpha_{k}}^{h, \delta}\right)-z_{\alpha_{k}}^{h, \delta}+\frac{\alpha_{k}}{c}\left(\tilde{y}_{n, \alpha_{k}}^{h, \delta}-\tilde{x}_{0, \alpha_{k}}^{h, \delta}\right)\right]
$$

where $\tilde{x}_{0, \alpha_{k}}^{h, \delta}:=P_{h} x_{0}$. Note that with the above notation

$$
\left\|R\left(\tilde{x}_{n, \alpha_{k}}^{h, \delta}\right)^{-1} P_{h} F^{\prime}\left(\tilde{x}_{n, \alpha_{k}}^{h, \delta}\right)\right\| \leqslant 1+\tau_{0} .
$$

Let

$$
\tilde{e}_{n, \alpha_{k}}^{h, \delta}:=\left\|\tilde{y}_{n, \alpha_{k}}^{h, \delta}-\tilde{x}_{n, \alpha_{k}}^{h, \delta}\right\|, \quad \forall n \geqslant 0
$$

and suppose that

$$
0<K_{0}<\frac{1}{4\left(1+\tau_{0}\right)}
$$

and

$$
\frac{\delta_{0}+\varepsilon_{0}}{\sqrt{\alpha_{0}}}<\frac{2}{2 M+3} \text {. }
$$

Let $\left\|\hat{x}-x_{0}\right\| \leqslant \rho$, with

$$
\rho<\frac{1}{M}\left(1-\left(\frac{3}{2}+M\right) \frac{\delta_{0}+\varepsilon_{0}}{\sqrt{\alpha_{0}}}\right)
$$


and

$$
\tilde{\gamma}_{\rho}:=M \rho+\left(\frac{3}{2}+M\right)\left(\frac{\varepsilon_{0}+\delta_{0}}{\sqrt{\alpha_{0}}}\right),
$$

and let $r$ be such that

$$
\tilde{r} \in\left(\tilde{r}_{1}, \tilde{r}_{2}\right)
$$

where

$$
\tilde{r}_{1}=\frac{1+\sqrt{1-16\left(1+\tau_{0}\right) K_{0} \tilde{\gamma}_{\rho}}}{8\left(1+\tau_{0}\right)}
$$

and

$$
\begin{aligned}
& \tilde{r}_{2}=\frac{1-\sqrt{1-16\left(1+\tau_{0}\right) K_{0} \tilde{\gamma}_{\rho}}}{8\left(1+\tau_{0}\right)} \\
& \tilde{b}:=4\left(1+\tau_{0}\right) K_{0} \tilde{r} .
\end{aligned}
$$

Then we have by (3.28)-(3.32) that

$$
0<\tilde{\gamma}_{\rho}<\frac{1}{16\left(1+\tau_{0}\right) K_{0}}
$$

Theorem 3.11. Let $\tilde{e}_{n, \alpha_{k}}^{h, \delta}$ be as in equation (3.27) with $\delta \in\left(0, \delta_{0}\right], \alpha=\alpha_{k}$ and $\varepsilon_{h} \in\left(0, \varepsilon_{0}\right]$. Then by Assumption 3.2 the following hold:

(a)

$$
\begin{aligned}
\left\|\tilde{x}_{n, \alpha_{k}}^{h, \delta}-\tilde{y}_{n-1, \alpha_{k}}^{h, \delta}\right\| \leqslant & \frac{K_{0}}{2}\left(1+\tau_{0}\right)\left[3\left\|\tilde{x}_{n-1, \alpha_{k}}^{h, \delta}-\tilde{x}_{0, \alpha_{k}}^{h, \delta}\right\|\right. \\
& \left.+5\left\|\tilde{y}_{n-1, \alpha_{k}}^{h, \delta}-\tilde{x}_{0, \alpha_{k}}^{h, \delta}\right\|\right] e_{n-1, \alpha_{k}}^{h, \delta}
\end{aligned}
$$

(b)

$$
\begin{aligned}
\left\|\tilde{x}_{n, \alpha_{k}}^{h, \delta}-\tilde{x}_{n-1, \alpha_{k}}^{h, \delta}\right\| \leqslant & \left\{1+\frac{K_{0}}{2}\left(1+\tau_{0}\right)\left[3\left\|\tilde{x}_{n-1, \alpha_{k}}^{h, \delta}-\tilde{x}_{0, \alpha_{k}}^{h, \delta}\right\|\right.\right. \\
& \left.\left.+5\left\|\tilde{y}_{n-1, \alpha_{k}}^{h, \delta}-\tilde{x}_{0, \alpha_{k}}^{h, \delta}\right\|\right]\right\} e_{n-1, \alpha_{k}}^{h, \delta} .
\end{aligned}
$$

(c)

$$
\begin{aligned}
\tilde{e}_{n, \alpha_{k}}^{h, \delta} \leqslant & \frac{K_{0}}{2}\left(1+\tau_{0}\right)\left[5\left\|\tilde{x}_{n, \alpha_{k}}^{h, \delta}-\tilde{x}_{0, \alpha_{k}}^{h, \delta}\right\|\right. \\
& \left.+3\left\|\tilde{y}_{n-1, \alpha_{k}}^{h, \delta}-\tilde{x}_{0, \alpha_{k}}^{h, \delta}\right\|\right]\left\|\tilde{y}_{n-1, \alpha_{k}}^{h, \delta}-\tilde{x}_{n, \alpha_{k}}^{h, \delta}\right\| \\
\leqslant & \tilde{b}^{2} \tilde{e}_{n-1, \alpha_{k}}^{h, \delta} \leqslant \tilde{b}^{2 n} \tilde{e}_{0, \alpha_{k}}^{h, \delta} \leqslant \tilde{b}^{2 n} \gamma_{\rho} .
\end{aligned}
$$


154 Ioannis K. Argyros, Monnanda E. Shobha, Santhosh George

Proof. Observe that

$$
\begin{aligned}
\tilde{x}_{n, \alpha_{k}}^{h, \delta}-\tilde{y}_{n-1, \alpha_{k}}^{h, \delta}= & \tilde{y}_{n-1, \alpha_{k}}^{h, \delta}-\tilde{x}_{n-1, \alpha_{k}}^{h, \delta}-R\left(\tilde{y}_{n-1, \alpha_{k}}^{h, \delta}\right)^{-1} P_{h}\left(F\left(\tilde{y}_{n-1, \alpha_{k}}^{h, \delta}\right)-z_{\alpha_{k}}^{h, \delta}\right. \\
& \left.+\frac{\alpha_{k}}{c}\left(\tilde{y}_{n-1, \alpha_{k}}^{h, \delta}-x_{0}\right)\right)+R\left(\tilde{x}_{n-1, \alpha_{k}}^{h, \delta}\right)^{-1} P_{h}\left(F\left(\tilde{x}_{n-1, \alpha_{k}}^{h, \delta}\right)\right. \\
& \left.-z_{\alpha_{k}}^{h, \delta}+\frac{\alpha_{k}}{c}\left(\tilde{x}_{n-1, \alpha_{k}}^{h, \delta}-x_{0}\right)\right) \\
= & \tilde{y}_{n-1, \alpha_{k}}^{h, \delta}-\tilde{x}_{n-1, \alpha_{k}}^{h, \delta}-R\left(\tilde{y}_{n-1, \alpha_{k}}^{h, \delta}\right)^{-1} P_{h}\left[F\left(\tilde{y}_{n-1, \alpha_{k}}^{h, \delta}\right)\right. \\
& \left.-F\left(\tilde{x}_{n-1, \alpha_{k}}^{h, \delta}\right)+\frac{\alpha_{k}}{c}\left(\tilde{y}_{n-1, \alpha_{k}}^{h, \delta}-\tilde{x}_{n-1, \alpha_{k}}^{h, \delta}\right)\right]+\left[R\left(\tilde{x}_{n-1, \alpha_{k}}^{h, \delta}\right)^{-1}\right. \\
& \left.-R\left(\tilde{y}_{n-1, \alpha_{k}}^{h, \delta}\right)^{-1}\right]\left(F\left(\tilde{x}_{n-1, \alpha_{k}}^{h, \delta}\right)-z_{\alpha_{k}}^{h, \delta}+\frac{\alpha_{k}}{c}\left(\tilde{x}_{n-1, \alpha_{k}}^{h, \delta}-x_{0}\right)\right) \\
= & R\left(\tilde{y}_{n-1, \alpha_{k}}^{h, \delta}\right)^{-1} P_{h}\left[F^{\prime}\left(\tilde{y}_{n-1, \alpha_{k}}^{h, \delta}\right)\left(\tilde{y}_{n-1, \alpha_{k}}^{h, \delta}-\tilde{x}_{n-1, \alpha_{k}}^{h, \delta}\right)\right. \\
& -\left(F\left(\tilde{y}_{n-1, \alpha_{k}}^{h, \delta}\right)-F\left(\tilde{x}_{n-1, \alpha_{k}}^{h, \delta}\right)\right]-R\left(\tilde{y}_{n-1, \alpha_{k}}^{h, \delta}\right)^{-1} \\
& \times\left[F^{\prime}\left(\tilde{y}_{n-1, \alpha_{k}}^{h, \delta}\right)-F^{\prime}\left(\tilde{x}_{n-1, \alpha_{k}}^{h, \delta}\right)\right]\left(\tilde{y}_{n-1, \alpha_{k}}^{h, \delta}-\tilde{x}_{n-1, \alpha_{k}}^{h, \delta}\right) \\
= & R\left(\tilde{y}_{n-1, \alpha_{k}}^{h, \delta}\right)^{-1} P_{h} \int_{0}^{1}\left[F^{\prime}\left(\tilde{y}_{n-1, \alpha_{k}}^{h, \delta}\right)-F^{\prime}\left(\tilde{x}_{n-1, \alpha_{k}}^{h, \delta}+t\left(\tilde{y}_{n-1, \alpha_{k}}^{h, \delta}\right.\right.\right. \\
& \left.\left.-\tilde{x}_{n-1, \alpha_{k}}^{h, \delta}\right)\right] P_{h}\left(\tilde{y}_{n-1, \alpha_{k}}^{h, \delta}-\tilde{x}_{n-1, \alpha_{k}}^{h, \delta}\right) d t-R\left(\tilde{y}_{n-1, \alpha_{k}}^{h, \delta}\right)^{-1} \\
& \times\left[F^{\prime}\left(\tilde{y}_{n-1, \alpha_{k}}^{h, \delta}\right)-F^{\prime}\left(\tilde{x}_{n-1, \alpha_{k}}^{h, \delta}\right)\right]\left(\tilde{y}_{n-1, \alpha_{k}}^{h, \delta}-\tilde{x}_{n-1, \alpha_{k}}^{h, \delta}\right)
\end{aligned}
$$

Now by Assumption 3.2 and (3.26) we have

$$
\begin{aligned}
\left\|\tilde{x}_{n, \alpha_{k}}^{h, \delta}-\tilde{y}_{n-1, \alpha_{k}}^{h, \delta}\right\| \leqslant & \left(1+\tau_{0}\right)\left[\| \int_{0}^{1} \Phi\left(\tilde{y}_{n-1, \alpha_{k}}^{h, \delta}, \tilde{x}_{n-1, \alpha_{k}}^{h, \delta}+t\left(\tilde{y}_{n-1, \alpha_{k}}^{h, \delta}\right.\right.\right. \\
& \left.\left.-\tilde{x}_{n-1, \alpha_{k}}^{h, \delta}\right), \tilde{y}_{n-1, \alpha_{k}}^{h, \delta}-\tilde{x}_{n-1, \alpha_{k}}^{h, \delta}\right) d t \| \\
& \left.+\left\|\Phi\left(\tilde{y}_{n-1, \alpha_{k}}^{h, \delta}, \tilde{x}_{n-1, \alpha_{k}}^{h, \delta}, \tilde{y}_{n-1, \alpha_{k}}^{h, \delta}-\tilde{x}_{n-1, \alpha_{k}}^{h, \delta}\right)\right\|\right] .
\end{aligned}
$$

The remaining part of the proof is analogous to the proof of Theorem 3.4.

Theorem 3.12. Let $\tilde{r}$ be as defined in (3.31) and the assumptions of Theorem 3.11 hold. Then $\tilde{x}_{n, \alpha_{k}}^{h, \delta}, \tilde{y}_{n, \alpha_{k}}^{h, \delta} \in B_{\tilde{r}}\left(P_{h} x_{0}\right)$, for all $n \geqslant 0$.

Proof. The proof is analogous to the proof of Theorem 3.5.

Theorem 3.13. Let $\tilde{y}_{n, \alpha_{k}}^{h, \delta}$ and $\tilde{x}_{n, \alpha_{k}}^{h, \delta}$ be as in (3.23) and (3.24) respectively and hypotheses of Theorem 3.12 hold. Then $\left(\tilde{x}_{n, \alpha_{k}}^{h, \delta}\right)$ is a Cauchy sequence in $B_{\tilde{r}}\left(P_{h} x_{0}\right)$ and converges to $x_{c, \alpha_{k}}^{h, \delta} \in \overline{B_{\tilde{r}}\left(P_{h} x_{0}\right)}$. Further $P_{h}\left[F\left(x_{c, \alpha_{k}}^{h, \delta}\right)+\frac{\alpha_{k}}{c}\left(x_{c, \alpha_{k}}^{h, \delta}-x_{0}\right)\right]=P_{h} z_{\alpha_{k}}^{h, \delta}$ and

$$
\left\|\tilde{x}_{n, \alpha_{k}}^{h, \delta}-x_{c, \alpha_{k}}^{h, \delta}\right\| \leqslant \frac{(1+\tilde{b}) \tilde{b}^{2 n} \tilde{\gamma}_{\rho}}{1-\tilde{b}^{2}}
$$

where $\tilde{\gamma}_{\rho}$ and $\tilde{b}$ are defined by (3.30) and (3.32), respectively. 
Proof. Using the relation (b) and (c) of Theorem 3.11, one can show that $\left(\tilde{x}_{n, \alpha_{k}}^{h, \delta}\right)$ is a Cauchy sequence in $B_{\tilde{r}}\left(P_{h} x_{0}\right)$. Observe that from (3.23)

$$
\begin{aligned}
\left\|P_{h}\left(F\left(\tilde{x}_{n, \alpha_{k}}^{h, \delta}\right)-z_{\alpha_{k}}^{h, \delta}\right)+\frac{\alpha_{k}}{c}\left(\tilde{x}_{n, \alpha_{k}}^{h, \delta}-P_{h} x_{0}\right)\right\| & =\left\|R\left(\tilde{x}_{n, \alpha_{k}}^{h, \delta}\right)\left(\tilde{x}_{n, \alpha_{k}}^{h, \delta}-\tilde{y}_{n, \alpha_{k}}^{h, \delta}\right)\right\| \\
& \leqslant\left\|R\left(\tilde{x}_{n, \alpha_{k}}^{h, \delta}\right)\right\|\left\|\tilde{y}_{n, \alpha_{k}}^{h, \delta}-\tilde{x}_{n, \alpha_{k}}^{h, \delta}\right\| \\
& \leqslant\left(\left\|P_{h} F^{\prime}\left(\tilde{x}_{n, \alpha_{k}}^{h, \delta}\right) P_{h}\right\|+\frac{\alpha_{k}}{c}\right) \tilde{e}_{n, \alpha_{k}}^{h, \delta} \\
& \leqslant\left(M+\frac{\alpha_{k}}{c}\right) \tilde{e}_{n, \alpha_{k}}^{h, \delta} .
\end{aligned}
$$

Now by letting $n \rightarrow \infty$ in $(3.36)$ we obtain $P_{h} F\left(x_{c, \alpha_{k}}^{h, \delta}\right)+\frac{\alpha_{k}}{c}\left(x_{c, \alpha_{k}}^{h, \delta}-P_{h} x_{0}\right)=P_{h} z_{\alpha_{k}}^{h, \delta}$. This completes the proof.

\section{Remark 3.14.}

(a) The convergence order of (TSNTM) would be four under Assumption 3.1. In Theorem 3.6 and 3.13 the error bounds are too pessimistic. That is why in practice we shall use the computational order of convergence (COC) (see eg. [6]) defined by

$$
\varrho \approx \ln \left(\frac{\left\|x_{n+1}-x_{\alpha}^{\delta}\right\|}{\left\|x_{n}-x_{\alpha}^{\delta}\right\|}\right) / \ln \left(\frac{\left\|x_{n}-x_{\alpha}^{\delta}\right\|}{\left\|x_{n-1}-x_{\alpha}^{\delta}\right\|}\right) .
$$

The (COC) $\varrho$ will then be close to 4 which is the order of convergence of (TSNTM).

Hereafter we assume that $\tilde{r}<\frac{1}{K_{0}}$ and $K_{1}<\frac{1-K_{0} \tilde{r}}{1-c}$.

We quote the following Theorems for our further analysis, whose proofs are given in [11].

Theorem 3.15 (see [11, Theorem 3.7]). Suppose $x_{c, \alpha_{k}}^{\delta}$ is the solution of

$$
F(x)+\frac{\alpha_{k}}{c}\left(x-x_{0}\right)=z_{\alpha_{k}}^{\delta}
$$

and Assumption 3.2 and 3.10 hold. Then

$$
\left\|\hat{x}-x_{c, \alpha_{k}}^{\delta}\right\| \leqslant \frac{\varphi_{1}\left(\alpha_{k}\right)+\left(2+\frac{4 \mu}{\mu-1}\right) \mu \psi^{-1}\left(\delta+\varepsilon_{h}\right)}{1-(1-c) K_{1}-K_{0} \tilde{r}} .
$$

Theorem 3.16 (see [11, Theorem 3.8]). Suppose $x_{c, \alpha_{k}}^{h, \delta}$ is the solution of (3.22) and Assumption 2.1 and Theorem 3.15 hold. In addition if $\tau_{0}<1$, then

$$
\left\|x_{c, \alpha_{k}}^{h, \delta}-x_{c, \alpha_{k}}^{\delta}\right\| \leqslant \frac{2}{1-\tau_{0}}\left(\frac{\delta+\varepsilon_{h}}{\sqrt{\alpha_{k}}}\right) .
$$

Theorem 3.17. Let $\tilde{x}_{n, \alpha_{k}}^{h, \delta}$ be as in (3.24), assumptions in Theorem 3.13, Theorem 3.15 and Theorem 3.16 hold. Then

$$
\left\|\hat{x}-\tilde{x}_{n, \alpha_{k}}^{h, \delta}\right\| \leqslant \frac{(1+\tilde{b}) \tilde{b}^{2 n} \tilde{\gamma}_{\rho}}{1-\tilde{b}^{2}}+\frac{\varphi_{1}\left(\alpha_{k}\right)+\left(2+\frac{4 \mu}{\mu-1}\right) \mu \psi^{-1}\left(\delta+\varepsilon_{h}\right)}{1-(1-c) K_{1}-K_{0} \tilde{r}}+\frac{2}{1-\tau_{0}}\left(\frac{\delta+\varepsilon_{h}}{\sqrt{\alpha_{k}}}\right) .
$$


Theorem 3.18. Let $\tilde{x}_{n, \alpha_{k}}^{h, \delta}$ be as in (3.24) and assumptions in Theorem 3.17 hold. Further let $\varphi_{1}\left(\alpha_{k}\right) \leqslant \varphi\left(\alpha_{k}\right)$ and

$$
n_{k}:=\min \left\{n: \tilde{b}^{2 n} \leqslant \frac{\delta+\varepsilon_{h}}{\sqrt{\alpha_{k}}}\right\} .
$$

Then

$$
\left\|\hat{x}-\tilde{x}_{n, \alpha_{k}}^{h, \delta}\right\|=O\left(\psi^{-1}\left(\delta+\varepsilon_{h}\right)\right) .
$$

\section{Algorithm}

Note that for $i, j \in\{0,1,2, \cdots, N\}$,

$z_{\alpha_{i}}^{h, \delta}-z_{\alpha_{j}}^{h, \delta}=\left(\alpha_{j}-\alpha_{i}\right)\left(P_{h} K^{*} K P_{h}+\alpha_{j} I\right)^{-1}\left(P_{h} K^{*} K P_{h}+\alpha_{i} I\right)^{-1} P_{h} K^{*}\left(f^{\delta}-K F\left(x_{0}\right)\right)$.

Therefore the balancing principle algorithm associated with the choice of the parameter specified in section 2 involves the following steps.

Step 1: Choose $\alpha_{0}$ such that $\delta_{0}+\varepsilon_{0}<\frac{2 \sqrt{\alpha_{0}}}{\beta(2 M+3)}, \mu>\left\{1, \frac{\beta(2 M+3)}{2}\right\}$ for Case 1 and $\delta_{0}+\varepsilon_{0}<\frac{2 \sqrt{\alpha_{0}}}{2 M+3}$ and $\mu>1$ for Case 2 ;

Step 2: $\alpha_{i}=\mu^{2 i} \alpha_{0}$;

Step 3: solve for $w_{i}$ :

$$
\left(P_{h} K^{*} K P_{h}+\alpha_{i} I\right) w_{i}=P_{h} K^{*}\left(f^{\delta}-K F\left(x_{0}\right)\right) ;
$$

Step 4: solve for $j<i, z_{i j}^{h, \delta}: \quad\left(P_{h} K^{*} K P_{h}+\alpha_{j} I\right) z_{i j}^{h, \delta}=\left(\alpha_{j}-\alpha_{i}\right) w_{i}$;

Step 5: if $\left\|z_{i j}^{h, \delta}\right\|>\frac{4 C\left(\delta+\varepsilon_{h}\right)}{\sqrt{\alpha_{j}}}$, then take $k=i-1$;

Step 6: otherwise, repeat with $i+1$ in place of $i$.

Step \%: choose $n_{k}:=\min \left\{n: b^{2 n} \leqslant \frac{\delta+\varepsilon_{h}}{\sqrt{\alpha_{k}}}\right\}$ in Section 3.1 and $n_{k}:=\min \{n:$ $\left.\tilde{b}^{2 n} \leqslant \frac{\delta+\varepsilon_{h}}{\sqrt{\alpha_{k}}}\right\}$ in Section 3.2

Step 8: solve $x_{n_{k}, \alpha_{k}}^{h, \delta}$ using the iteration (3.3) or $\tilde{x}_{n_{k}, \alpha_{k}}^{h, \delta}$ using the iteration (3.24).

\section{Implementation of the method}

Let $V_{n}$ be a sequence of finite dimensional subspaces of $X$ and let $P_{h}=P_{\frac{1}{n}}$ denote the orthogonal projection on $X$ with range $R\left(P_{h}\right)=V_{n}$. We assume that $\operatorname{dim} V_{n}=n+1$ and $\left\|P_{h} x-x\right\| \rightarrow 0$ as $h \rightarrow 0$ for all $x \in X$. We choose the linear splines $\left\{v_{1}, v_{2}, \cdots, v_{n+1}\right\}$ in a uniform grid of $n+1$ points in $[0,1]$ as a basis of $V_{n}$.

Since $w_{i} \in V_{n}, w_{i}$ is of the form $\sum_{i=1}^{n+1} \lambda_{i} v_{i}$ for some scalars $\lambda_{1}, \lambda_{2}, \cdots, \lambda_{n+1}$. It can be seen that $w_{i}$ is a solution of (4.1) if and only if $\bar{\lambda}=\left(\lambda_{1}, \lambda_{2}, \cdots, \lambda_{n+1}\right)^{T}$ is the unique solution of

$$
\left(M_{n}+\alpha_{i} B_{n}\right) \bar{\lambda}=\bar{a}
$$


where

$$
\begin{aligned}
M_{n} & =\left(\left\langle K v_{i}, K v_{j}\right\rangle\right), \quad i, j=1,2, \cdots, n+1 \\
B_{n} & =\left(\left\langle v_{i}, v_{j}\right\rangle\right), \quad i, j=1,2, \cdots, n+1
\end{aligned}
$$

and

$$
\bar{a}=\left(\left\langle P_{h} K^{*}\left(f^{\delta}-K F\left(x_{0}\right)\right), v_{i}\right\rangle\right)^{T}, \quad i=1,2, \cdots, n+1 .
$$

Observe that $z_{i j}^{h, \delta}$ in step 4 of algorithm is again in $V_{n}$ and hence $z_{i j}^{h, \delta}=$ $\sum_{k=1}^{n+1} \mu_{k}^{i j} v_{k}$ for some $\mu_{k}^{i j}, k=1,2, \cdots, n+1$. One can see that for $j<i, z_{i j}^{h, \delta}$ is a solution of

$$
\left(P_{h} K^{*} K P_{h}+\alpha_{j} I\right) z_{i j}^{h, \delta}=\left(\alpha_{j}-\alpha_{i}\right) w_{i}
$$

if and only if $\overline{\mu^{i j}}=\left(\mu_{1}^{i j}, \mu_{2}^{i j}, \cdots, \mu_{n+1}^{i j}\right)^{T}$ is the unique solution of

$$
\left(M_{n}+\alpha_{j} B_{n}\right) \overline{\mu^{i j}}=\bar{b}
$$

where

$$
\bar{b}=\left(\left\langle\left(\alpha_{j}-\alpha_{i}\right) w_{i}, v_{i}\right\rangle\right)^{T} .
$$

Compute $z_{i j}^{h, \delta}$ till $\left\|z_{i j}^{h, \delta}\right\|>\frac{4 C\left(\delta+\varepsilon_{h}\right)}{\sqrt{\alpha_{j}}}$ and fix $k=i-1$. Now choose $n_{k}=\min \{n$ : $\left.b^{2 n} \leqslant \frac{\delta+\varepsilon_{h}}{\sqrt{\alpha_{k}}}\right\}$ for Case 1 and $n_{k}=\min \left\{n: \tilde{b}^{2 n} \leqslant \frac{\delta+\varepsilon_{h}}{\sqrt{\alpha_{k}}}\right\}$ for Case 2.

Case 1: Since $y_{n_{k}, \alpha_{k}}^{h, \delta}, x_{n_{k}, \alpha_{k}}^{h, \delta} \in V_{n}$, let $y_{n_{k}, \alpha_{k}}^{h, \delta}=\sum_{i=1}^{n+1} \xi_{i}^{n} v_{i}$ and $x_{n_{k}, \alpha_{k}}^{h, \delta}=$ $\sum_{i=1}^{n+1} \eta_{i}^{n} v_{i}$, where $\xi_{i}^{n}$ and $\eta_{i}^{n}$ are some scalars. Then from (3.2) we have

$$
P_{h} F^{\prime}\left(x_{n_{k}, \alpha_{k}}^{h, \delta}\right)\left(y_{n_{k}, \alpha_{k}}^{h, \delta}-x_{n_{k}, \alpha_{k}}^{h, \delta}\right)=P_{h}\left[z_{\alpha_{k}}^{h, \delta}-F\left(x_{n_{k}, \alpha_{k}}^{h, \delta}\right)\right] .
$$

Observe that $\left(y_{n_{k}, \alpha_{k}}^{h, \delta}-x_{n_{k}, \alpha_{k}}^{h, \delta}\right)$ is a solution of (5.1) if and only if $\left(\overline{\xi^{n}-\eta^{n}}\right)=$ $\left(\xi_{1}^{n}-\eta_{1}^{n}, \xi_{2}^{n}-\eta_{2}^{n}, \cdots, \xi_{n+1}^{n}-\eta_{n+1}^{n}\right)^{T}$ is the unique solution of

$$
Q_{n}\left(\overline{\xi^{n}-\eta^{n}}\right)=B_{n}\left[\overline{\lambda^{n}}-F_{h 1}\right]
$$

where

$$
\begin{aligned}
Q_{n} & =\left\langle F^{\prime}\left(x_{n_{k}, \alpha_{k}}^{h, \delta}\right) v_{i}, v_{j}\right\rangle, \quad i, j=1,2, \cdots, n+1, \\
F_{h 1} & =\left[F\left(x_{n_{k}, \alpha_{k}}^{h, \delta}\right)\left(t_{1}\right), F\left(x_{n_{k}, \alpha_{k}}^{h, \delta}\right)\left(t_{2}\right), \cdots, F\left(x_{n_{k}, \alpha_{k}}^{h, \delta}\right)\left(t_{n+1}\right)\right]^{T},
\end{aligned}
$$

where $t_{1}, t_{2}, \cdots, t_{n+1}$ are the grid points.

Further from (3.3) it follows that

$$
P_{h} F^{\prime}\left(y_{n_{k}, \alpha_{k}}^{h, \delta}\right)\left(x_{n_{k}+1, \alpha_{k}}^{h, \delta}-y_{n_{k}, \alpha_{k}}^{h, \delta}\right)=P_{h}\left[z_{\alpha_{k}}^{h, \delta}-F\left(y_{n_{k}, \alpha_{k}}^{h, \delta}\right)\right] .
$$

Thus $\left(x_{n_{k}+1, \alpha_{k}}^{h, \delta}-y_{n_{k}, \alpha_{k}}^{h, \delta}\right)$ is a solution of (5.2) if and only if $\left(\overline{\eta^{n+1}-\xi^{n}}\right)=\left(\eta_{1}^{n+1}-\right.$ $\left.\xi_{1}^{n}, \eta_{2}^{n+1}-\xi_{2}^{n}, \cdots, \eta_{n+1}^{n+1}-\xi_{n+1}^{n}\right)^{T}$ is the unique solution of

$$
\tilde{Q}_{n}\left(\overline{\eta^{n+1}-\xi^{n}}\right)=B_{n}\left[\overline{\lambda^{n}}-F_{h 2}\right]
$$


where

$$
\begin{aligned}
\tilde{Q}_{n} & =\left\langle F^{\prime}\left(y_{n_{k}, \alpha_{k}}^{h, \delta}\right) v_{i}, v_{j}\right\rangle, \quad i, j=1,2, \cdots, n+1, \\
F_{h 2} & =\left[F\left(y_{n_{k}, \alpha_{k}}^{h, \delta}\right)\left(t_{1}\right), F\left(y_{n_{k}, \alpha_{k}}^{h, \delta}\right)\left(t_{2}\right), \cdots, F\left(y_{n_{k}, \alpha_{k}}^{h, \delta}\right)\left(t_{n+1}\right)\right]^{T} .
\end{aligned}
$$

Case 2: Let $\tilde{\xi}^{n}=\left(\tilde{\xi}_{1}^{n}, \tilde{\xi}_{2}^{n}, \cdots, \tilde{\xi}_{n+1}^{n}\right), \tilde{\eta}^{n}=\left(\tilde{\eta}_{1}^{n}, \tilde{\eta}_{2}^{n}, \cdots, \tilde{\eta}_{n+1}^{n}\right), \tilde{y}_{n, \alpha_{k}}^{h, \delta}=\sum_{i=1}^{n+1} \tilde{\xi}_{i}^{n} v_{i}$ and $\tilde{x}_{n, \alpha_{k}}^{h, \delta}=\sum_{i=1}^{n+1} \tilde{\eta}_{i}^{n} v_{i}$. Then from (3.23) we have

$$
\begin{aligned}
\left(P_{h} F^{\prime}\left(\tilde{x}_{n, \alpha_{k}}^{h, \delta}\right)+\frac{\alpha_{k}}{c}\right) \sum_{i=1}^{n+1}\left(\tilde{\xi}_{i}^{n}-\tilde{\eta}_{i}^{n}\right) v_{i}= & \sum_{i=1}^{n+1} \lambda_{i} v_{i}-\sum_{i=1}^{n+1} P_{h} F\left(\tilde{x}_{n, \alpha_{k}}^{h, \delta}\right) v_{i} \\
& +\frac{\alpha_{k}}{c} \sum_{i=1}^{n+1}\left(x_{0}\left(t_{i}\right)-\tilde{\eta}_{i}^{n}\right) v_{i}
\end{aligned}
$$

where $t_{1}, t_{2}, \cdots, t_{n+1}$ are the grid points.

Observe that $\left(\tilde{y}_{n, \alpha_{k}}^{h, \delta}-\tilde{x}_{n, \alpha_{k}}^{h, \delta}\right)$ is a solution of (3.23) if and only if $\left(\overline{\tilde{\xi}^{n}-\tilde{\eta}^{n}}\right)=$ $\left(\tilde{\xi}_{1}^{n}-\tilde{\eta}_{1}^{n}, \tilde{\xi}_{2}^{n}-\tilde{\eta}_{2}^{n}, \cdots, \tilde{\xi}_{n+1}^{n}-\tilde{\eta}_{n+1}^{n}\right)^{T}$ is the unique solution of

$$
\left(S_{n}+\frac{\alpha_{k}}{c} B_{n}\right)\left(\overline{\tilde{\xi}^{n}-\tilde{\eta}^{n}}\right)=B_{n}\left[\bar{\lambda}-F_{h 3}+\frac{\alpha_{k}}{c}\left(X_{0}-\overline{\tilde{\eta}^{n}}\right)\right],
$$

where

$$
\begin{aligned}
S_{n} & =\left\langle F^{\prime}\left(\tilde{x}_{n, \alpha_{k}}^{h, \delta}\right) v_{i}, v_{j}\right\rangle, \quad i, j=1,2, \cdots, n+1 \\
F_{h 3} & =\left[F\left(\tilde{x}_{n, \alpha_{k}}^{h, \delta}\right)\left(t_{1}\right), F\left(\tilde{x}_{n, \alpha_{k}}^{h, \delta}\right)\left(t_{2}\right), \cdots, F\left(\tilde{x}_{n, \alpha_{k}}^{h, \delta}\right)\left(t_{n+1}\right)\right]^{T}
\end{aligned}
$$

and $X_{0}=\left[x_{0}\left(t_{1}\right), x_{0}\left(t_{2}\right), \cdots, x_{0}\left(t_{n+1}\right)\right]^{T}$.

Further from (3.24) it follows that

$$
\left(P_{h} F^{\prime}\left(\tilde{y}_{n, \alpha_{k}}^{h, \delta}\right)+\frac{\alpha_{k}}{c}\right)\left(\tilde{x}_{n+1, \alpha_{k}}^{h, \delta}-\tilde{y}_{n, \alpha_{k}}^{h, \delta}\right)=P_{h}\left[z_{\alpha_{k}}^{h, \delta}-F\left(\tilde{y}_{n, \alpha_{k}}^{h, \delta}\right)+\frac{\alpha_{k}}{c}\left(x_{0, \alpha_{k}}^{h, \delta}-\tilde{y}_{n, \alpha_{k}}^{h, \delta}\right)\right] .
$$

Thus $\left(\tilde{x}_{n+1, \alpha_{k}}^{h, \delta}-\tilde{y}_{n, \alpha_{k}}^{h, \delta}\right)$ is a solution of (5.3) if and only if $\left.\overline{\left(\tilde{\eta}^{n+1}-\tilde{\xi}^{n}\right.}\right)=\left(\tilde{\eta}_{1}^{n+1}\right.$ $\left.\tilde{\xi}_{1}^{n}, \tilde{\eta}_{2}^{n+1}-\tilde{\xi}_{2}^{n}, \cdots, \tilde{\eta}_{n+1}^{n+1}-\tilde{\xi}_{n+1}^{n}\right)^{T}$ is the unique solution of

$$
\left(\tilde{S}_{n}+\frac{\alpha_{k}}{c} B_{n}\right)\left(\overline{\tilde{\eta}^{n+1}-\tilde{\xi}^{n}}\right)=B_{n}\left[\bar{\lambda}-F_{h 4}+\frac{\alpha_{k}}{c}\left(X_{0}-\overline{\tilde{\xi}^{n}}\right)\right],
$$

where

$$
\begin{aligned}
\tilde{S}_{n} & =\left\langle F^{\prime}\left(\tilde{y}_{n, \alpha_{k}}^{h, \delta}\right) v_{i}, v_{j}\right\rangle, \quad i, j=1,2, \cdots, n+1, \\
F_{h 4} & =\left[F\left(\tilde{y}_{n, \alpha_{k}}^{h, \delta}\right)\left(t_{1}\right), F\left(\tilde{y}_{n, \alpha_{k}}^{h, \delta}\right)\left(t_{2}\right), \cdots, F\left(\tilde{y}_{n, \alpha_{k}}^{h, \delta}\right)\left(t_{n+1}\right)\right]^{T} .
\end{aligned}
$$

Example 5.1. To illustrate the method for Case 1, we consider the operator $K F: L^{2}(0,1) \longrightarrow L^{2}(0,1)$ where $F: D(F) \subseteq L^{2}(0,1) \longrightarrow L^{2}(0,1)$ defined by

$$
F(u):=u^{3}
$$


and $K: L^{2}(0,1) \longrightarrow L^{2}(0,1)$ defined by

$$
K(x)(t)=\int_{0}^{1} k(t, s) x(s) d s
$$

where

$$
k(t, s)= \begin{cases}(1-t) s, & 0 \leqslant s \leqslant t \leqslant 1 \\ (1-s) t, & 0 \leqslant t \leqslant s \leqslant 1\end{cases}
$$

The Fréchet derivative of $F$ is given by

$$
\begin{aligned}
F^{\prime}(u) w & =3\left(u^{2}\right) w, \\
{\left[F^{\prime}(v)-F^{\prime}(u)\right] w } & =3\left(v^{2}-u^{2}\right) w \\
& =3 u^{2}\left(\frac{v^{2}}{u^{2}}-1\right) w \\
& =F^{\prime}(u) \Phi(u, v, w),
\end{aligned}
$$

where $\Phi(u, v, w)=\left(\frac{v^{2}}{u^{2}}-1\right) w=\frac{(v+u)(v-u)}{u^{2}} w$. Thus $F$ satisfies the Assumption 3.2 with $k_{0} \geqslant\left\|\frac{(v+u)}{u^{2}}\right\|$.

We take $y(t)=\frac{-1}{144 \pi^{2}}\left[-54+63 \pi^{2} t^{2}-220 \sin (\pi t)+16 \sin (\pi t) \cos ^{2}(\pi t)+\right.$ $\left.54 \cos ^{2}(\pi t)-63 \pi^{2} t\right]$ and $y^{\delta}=y+\delta$. Then the exact solution

$$
\hat{x}(t)=1 / 2+\sin \pi t
$$

We use

$$
x_{0}(t)=\sin \pi t+3 / 5
$$

as our initial guess, then

$$
F\left(x_{0}\right)-F(\hat{x})=x_{0}^{3}-\hat{x}^{3} .
$$

Even though we are unable to write $F\left(x_{0}\right)-F(\hat{x})=\varphi\left(K^{*} K\right) w$ for some function $\varphi$, we use the function $\varphi(\lambda)=\lambda$ and obtain the results as given in the last column of the Table 1. Thus we expect to have an accuracy of order at least $O\left(\delta^{\frac{1}{2}}\right)$.

We choose $\alpha_{0}=(1.5)\left(\delta+\varepsilon_{h}\right)^{2}, \mu=1.3,\left(\delta+\varepsilon_{h}\right)=0.1, g\left(\gamma_{\rho}\right)=0.54$ approximately. In this example, for all $n$, the number of iteration $n_{k}=1$. The results of the computation are presented in Table 1 . The plots of the exact and the approximate solution obtained for $n=256$ to 1024 are given in Figure 1 .

Example 5.2. To illustrate the method for Case 2, we consider the operator $K F: L^{2}(0,1) \longrightarrow L^{2}(0,1)$ where $K: L^{2}(0,1) \longrightarrow L^{2}(0,1)$ defined by

$$
K(x)(t)=\int_{0}^{1} k(t, s) x(s) d s
$$


Table 1.

\begin{tabular}{|c|c|c|c|c|}
\hline $\mathrm{n}$ & $\mathrm{k}$ & $\alpha_{k}$ & $\left\|x_{k}-\hat{x}\right\|$ & $\frac{\left\|x_{k}-\hat{x}\right\|}{\delta^{1 / 2}}$ \\
\hline 8 & 4 & 0.1094 & 0.2199 & 0.6902 \\
\hline 16 & 4 & 0.1069 & 0.1645 & 0.5192 \\
\hline 32 & 4 & 0.1063 & 0.1342 & 0.4242 \\
\hline 64 & 4 & 0.1061 & 0.1178 & 0.3725 \\
\hline 128 & 4 & 0.1061 & 0.1091 & 0.3451 \\
\hline 256 & 4 & 0.1060 & 0.1046 & 0.3308 \\
\hline 512 & 4 & 0.1060 & 0.1023 & 0.3236 \\
\hline 1024 & 4 & 0.1060 & 0.1012 & 0.3199 \\
\hline
\end{tabular}

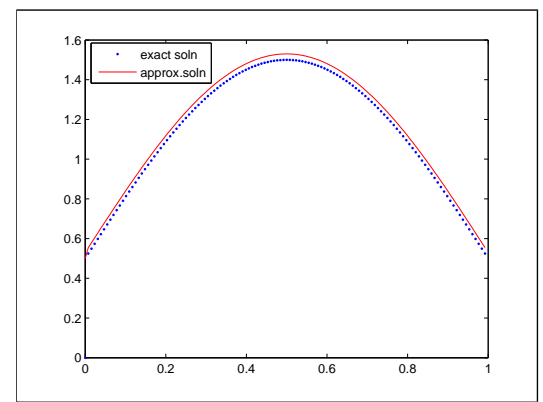

$\mathrm{n}=128$

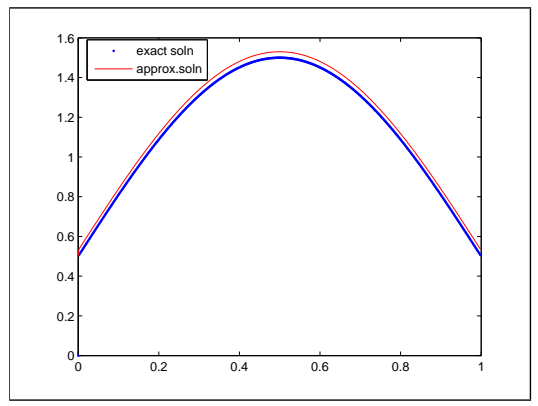

$\mathrm{n}=512$

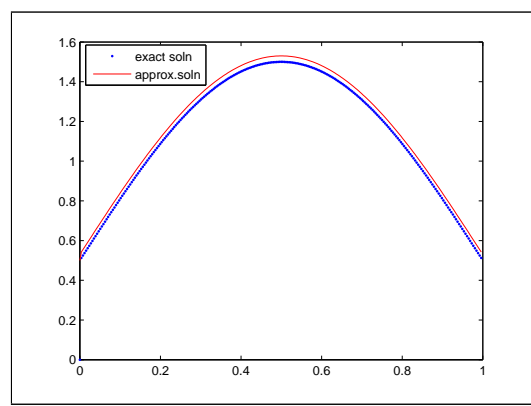

$\mathrm{n}=256$

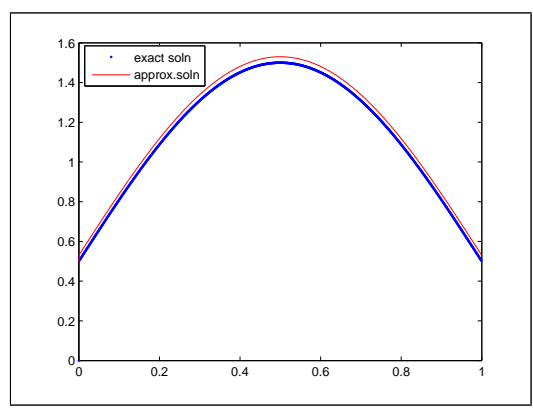

$\mathrm{n}=1024$

Figure 4. Curve of the exact and approximate solutions of Case 1

and $F: D(F) \subseteq L^{2}(0,1) \longrightarrow L^{2}(0,1)$ defined by

$$
F(u):=\int_{0}^{1} k(t, s) u^{3}(s) d s
$$


where

$$
k(t, s)= \begin{cases}(1-t) s, & 0 \leqslant s \leqslant t \leqslant 1 \\ (1-s) t, & \leqslant t \leqslant s \leqslant 1\end{cases}
$$

Then for all $x(t), y(t): x(t)>y(t)$ :

$$
\begin{aligned}
\langle F(x)-F(y), x-y\rangle= & \int_{0}^{1}\left[\int_{0}^{1} k(t, s)\left(x^{3}-y^{3}\right)(s) d s\right] \\
& \times(x-y)(t) d t \geqslant 0 .
\end{aligned}
$$

Thus the operator $F$ is monotone. The Fréchet derivative of $F$ is given by

$$
F^{\prime}(u) w=3 \int_{0}^{1} k(t, s)(u(s))^{2} w(s) d s .
$$

So for any $u \in B_{r}\left(x_{0}\right), x_{0}^{2}(s) \geqslant k_{3}>0, \forall s \in(0,1)$, we have

$$
F^{\prime}(u) w=F^{\prime}\left(x_{0}\right) G\left(u, x_{0}\right) w
$$

where $G\left(u, x_{0}\right)=\left(\frac{u}{x_{0}}\right)^{2}$.

Further observe that for $u(s)>0, \forall s \in(0,1)$,

$$
\begin{aligned}
{\left[F^{\prime}(v)-F^{\prime}(u)\right] w(s) } & =3 \int_{0}^{1} k(t, s) u^{2}(s)\left[\frac{\left(v^{2}(s)-u^{2}(s)\right) w(s)}{u^{2}(s)}\right] d s \\
: & =F^{\prime}(u) \Phi(u, v, w),
\end{aligned}
$$

where $\Phi(u, v, w)=\frac{\left(v^{2}(s)-u^{2}(s)\right) w(s)}{u^{2}(s)}$.

Note that

$$
\Phi(u, v, w)=\frac{(v(s)+u(s))(v(s)-u(s)) w(s)}{u^{2}(s)} .
$$

Thus $F$ satisfies the Assumption 3.2 with

$$
K_{0} \geqslant\left\|\frac{(v(s)+u(s))}{u^{2}(s)}\right\|
$$


In our computation, we take

$$
\begin{aligned}
f(t)= & \left(\frac{1}{18 \pi^{2}}\right)(1-t)\left(14 t-7+\cos ^{3}(\pi t)\right. \\
& +6 \cos (\pi t)) t^{2}-\left(\frac{1}{18 \pi^{2}}\right) t\left(14 t-7+\cos ^{3}(\pi t)\right. \\
& +6 \cos (\pi t))\left(1-t^{2}\right)+\left(\frac{1}{9 \pi^{2}}\right) t(1-t)\left(14 t-7+\cos ^{3}(\pi t)+6 \cos (\pi t)\right)
\end{aligned}
$$

and $f^{\delta}=f+\delta$. Then the exact solution

$$
\hat{x}(t)=\cos \pi t .
$$

We use

$$
\begin{aligned}
x_{0}(t)= & \cos (\pi t)+3\left[\frac { - 1 } { 4 \pi ^ { 2 } } \left(1-t+2 \pi t^{2} \cos (\pi t)\right.\right. \\
& \times \sin (\pi t)+\pi^{2} t^{3}+t \cos ^{2}(\pi t)-2 \pi t \cos (\pi t) \\
& \left.\times \sin (\pi t)-\pi^{2} t^{2}-\cos ^{2}(\pi t)\right)+\frac{1}{4 \pi^{2}} t \\
& \times\left(-2 \cos (\pi t) \sin (\pi t) \pi-2 \pi^{2} t+2 \pi t \cos (\pi t)\right. \\
& \left.\left.\times \sin (\pi t)+\pi^{2} t^{2}+\cos ^{2}(\pi t)+\pi^{2}-\cos ^{2}(\pi t)\right)\right]
\end{aligned}
$$

as our initial guess, so that the function $x_{0}-\hat{x}$ satisfies the source condition

$$
x_{0}-\hat{x}=\varphi_{1}\left(F^{\prime}\left(x_{0}\right)\right) 1
$$

where $\varphi_{1}(\lambda)=\lambda$. Thus we expect to have an accuracy of order at least $O\left(\delta^{\frac{1}{2}}\right)$.

We choose $\alpha_{0}=(1.3) \delta^{2}, \mu=1.3, \delta=0.1=c, \rho=0.19, \tilde{\gamma}_{\rho}=0.8173$ and $g\left(\tilde{\gamma}_{\rho}\right)=0.54$ approximately. For all $n$ the number of iteration $n_{k}=1$. The results of the computation are presented in Table 2. The plots of the exact and the approximate solution obtained for $n=128$ to 1024 are given in Figure 2.

Next we present two examples where Assumption 3.1 is not satisfied but 3.2 is satisfied.

Example 5.3. Let $X=Y=\mathbb{R}, D=[0, \infty), x_{0}=1$ and define function $F$ on $D$ by

$$
F(x)=\frac{x^{1+\frac{1}{i}}}{1+\frac{1}{i}}+c_{1} x+c_{2},
$$

where $c_{1}, c_{2}$ are real parameters and $i>2$ an integer. Then $F^{\prime}(x)=x^{1 / i}+c_{1}$ is not Lipschitz on $D$. However central Lipschitz condition Assumption 3.2 holds for $K_{0}=2$. 
Table 2.

\begin{tabular}{|c|c|c|c|c|c|}
\hline$n$ & $k$ & $\delta$ & $\alpha$ & $\left\|\tilde{x}_{k}-\hat{x}\right\|$ & $\frac{\left\|\tilde{x}_{k}-\hat{x}\right\|}{(\delta)^{1 / 2}}$ \\
\hline 8 & 4 & 0.1016 & 0.1094 & 0.3652 & 1.1458 \\
\hline 16 & 4 & 0.1004 & 0.1069 & 0.2664 & 0.8408 \\
\hline 32 & 4 & 0.1001 & 0.1063 & 0.1994 & 0.6303 \\
\hline 64 & 4 & 0.1000 & 0.1061 & 0.1554 & 0.4914 \\
\hline 128 & 4 & 0.1000 & 0.1061 & 0.1278 & 0.4042 \\
\hline 256 & 4 & 0.1000 & 0.1060 & 0.1115 & 0.3526 \\
\hline 512 & 4 & 0.1000 & 0.1060 & 0.1024 & 0.3238 \\
\hline 1024 & 4 & 0.1000 & 0.1060 & 0.0975 & 0.3083 \\
\hline
\end{tabular}

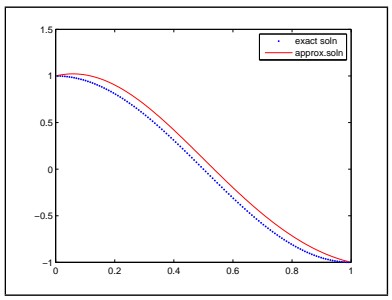

$n=128$

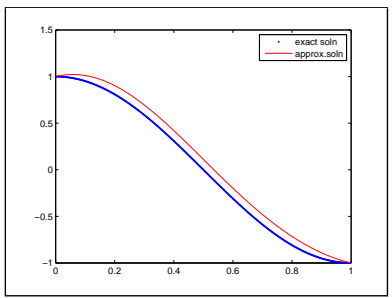

$n=512$

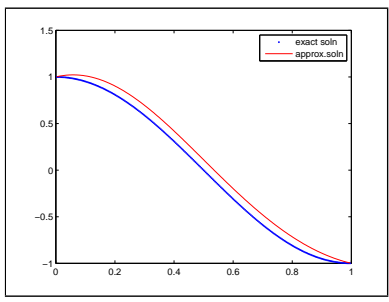

$n=256$

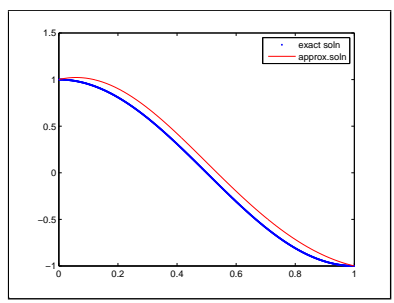

$n=1024$

Figure 5. Curve of the exact and approximate solutions of Case 2

Indeed, we have

$$
\left\|F^{\prime}(x)-F^{\prime}\left(x_{0}\right)\right\|=\left|x^{1 / i}-x_{0}^{1 / i}\right|=\frac{\left|x-x_{0}\right|}{x_{0}^{\frac{i-1}{i}}+\cdots+x^{\frac{i-1}{i}}}
$$

so

$$
\left\|F^{\prime}(x)-F^{\prime}\left(x_{0}\right)\right\| \leqslant 1\left|x-x_{0}\right| .
$$

Example 5.4. We consider the integral equations

$$
u(s)=f(s)+\lambda \int_{a}^{b} G(s, t) u(t)^{1+1 / n} d t, \quad n \in \mathbb{N} .
$$


Here, $f$ is a given continuous function satifying $f(s)>0, s \in[a, b], \lambda$ is a real number, and the kernel $G$ is continuous and positive in $[a, b] \times[a, b]$.

For example, when $G(s, t)$ is the Green kernel, the corresponding integral equation is equivalent to the boundary value problem

$$
\begin{aligned}
u^{\prime \prime} & =\lambda u^{1+1 / n} \\
u(a) & =f(a), u(b)=f(b) .
\end{aligned}
$$

These type of problems have been considered in [1]-[6].

Equation of the form (5.5) generalize equations of the form

$$
u(s)=\int_{a}^{b} G(s, t) u(t)^{n} d t
$$

studied in [1]-[6]. Instead of (5.5) we can try to solve the equation $F(u)=0$ where

$$
F: \Omega \subseteq C[a, b] \rightarrow C[a, b], \quad \Omega=\{u \in C[a, b]: u(s) \geqslant 0, s \in[a, b]\},
$$

and

$$
F(u)(s)=u(s)-f(s)-\lambda \int_{a}^{b} G(s, t) u(t)^{1+1 / n} d t .
$$

The norm we consider is the max-norm.

The derivative $F^{\prime}$ is given by

$$
F^{\prime}(u) v(s)=v(s)-\lambda\left(1+\frac{1}{n}\right) \int_{a}^{b} G(s, t) u(t)^{1 / n} v(t) d t, \quad v \in \Omega .
$$

First of all, we notice that $F^{\prime}$ does not satisfy a Lipschitz-type condition in $\Omega$. Let us consider, for instance, $[a, b]=[0,1], G(s, t)=1$ and $y(t)=0$. Then $F^{\prime}(y) v(s)=$ $v(s)$ and

$$
\left\|F^{\prime}(x)-F^{\prime}(y)\right\|=|\lambda|\left(1+\frac{1}{n}\right) \int_{a}^{b} x(t)^{1 / n} d t .
$$

If $F^{\prime}$ were a Lipschitz function, then

$$
\left\|F^{\prime}(x)-F^{\prime}(y)\right\| \leqslant L_{1}\|x-y\|
$$

or, equivalently, the inequality

$$
\int_{0}^{1} x(t)^{1 / n} d t \leqslant L_{2} \max _{x \in[0,1]} x(s)
$$

would hold for all $x \in \Omega$ and for a constant $L_{2}$. But this is not true. Consider, for example, the functions

$$
x_{j}(t)=\frac{t}{j}, \quad j \geqslant 1, t \in[0,1] .
$$

If these are substituted into (5.7)

$$
\frac{1}{j^{1 / n}(1+1 / n)} \leqslant \frac{L_{2}}{j} \Leftrightarrow j^{1-1 / n} \leqslant L_{2}(1+1 / n), \quad \forall j \geqslant 1 .
$$

This inequality is not true when $j \rightarrow \infty$. 
Therefore, condition (5.7) is not satisfied in this case. However, condition Assumption 3.2 holds. To show this, let $x_{0}(t)=f(t)$ and $\gamma=\min _{s \in[a, b]} f(s), \alpha>0$ Then for $v \in \Omega$,

$$
\begin{aligned}
\left\|\left[F^{\prime}(x)-F^{\prime}\left(x_{0}\right)\right] v\right\| & =|\lambda|\left(1+\frac{1}{n}\right) \max _{s \in[a, b]}\left|\int_{a}^{b} G(s, t)\left(x(t)^{1 / n}-f(t)^{1 / n}\right) v(t) d t\right| \\
& \leqslant|\lambda|\left(1+\frac{1}{n}\right) \max _{s \in[a, b]} G_{n}(s, t)
\end{aligned}
$$

where $G_{n}(s, t)=\frac{G(s, t)|x(t)-f(t)|}{x(t)^{(n-1) / n}+x(t)^{(n-2) / n} f(t)^{1 / n}+\cdots+f(t)^{(n-1) / n}}\|v\|$.

Hence,

$$
\begin{aligned}
\left\|\left[F^{\prime}(x)-F^{\prime}\left(x_{0}\right)\right] v\right\| & =\frac{|\lambda|(1+1 / n)}{\gamma^{(n-1) / n}} \max _{s \in[a, b]} \int_{a}^{b} G(s, t) d t\left\|x-x_{0}\right\| \\
& \leqslant \bar{K}_{0}\left\|x-x_{0}\right\|,
\end{aligned}
$$

where $\bar{K}_{0}=\frac{|\lambda|(1+1 / n)}{\gamma^{(n-1) / n}} N, K_{0}=2 \bar{K}_{0}$ and $N=\max _{s \in[a, b]} \int_{a}^{b} G(s, t) d t$. Then condition Assumption 3.2 holds for sufficiently small $\lambda$.

Acknowledgement. Ms. Shobha, thanks National Institute of Technology Karnataka, India, for the financial support.

\section{References}

[1] I.K. Argyros, Convergenve and Applications of Newton-type Iterations, Springer, New York, 2008.

[2] I.K. Argyros, Approximating solutions of equations using Newton's method with a modified Newton's method iterate as a starting point, Rev. Anal. Numer. Theor. Approx. 36 (2007), 123-138.

[3] I.K. Argyros, A Semilocal convergence for directional Newton methods, Math.Comput.(AMS). 80 (2011), 327-343.

[4] I.K. Argyros and S. Hilout, Weaker conditions for the convergence of Newton's method, J. Complexity 28 (2012), 364-387.

[5] I.K.Argyros, and Said Hilout(2010), A convergence analysis for directional two-step Newton methods, Numer. Algor. 55, 503-528.

[6] I.K. Argyros, Y.J. Cho and S. Hilout, Numerical methods for equations and its applications, CRC Press, Taylor and Francis, New York, 2012.

[7] S. George, Newton-Tikhonov regularization of ill-posed Hammerstein operator equation, J. Inverse and Ill-Posed Problems 2 (2006), 14, 135-146.

[8] S. George and M. Kunhanandan, An iterative regularization method for Illposed Hammerstein type operator equation, J. Inv. Ill-Posed Problems 17 (2009), 831-844.

[9] S. George and M.T. Nair, A modified Newton-Lavrentiev regularization for nonlinear ill-posed Hammerstein-Type operator equation, Journal of Complexity 24 (2008), 228-240. 
[10] S. George and M.E. Shobha, On Improving the Semilocal Convergence of Newton-Type Iterative method for Ill-posed Hammerstein type operator equations, IAENG-International Journal of Applied Mathematics 43 (2013), no. 2, $64-70$.

[11] S. George and M.E. Shobha, Two-Step Newton-Tikhonov Method for Hammerstein-Type Equations: Finite-Dimensional Realization, ISRN Applied Mathematics, vol. 2012, Article ID 783579, 22 pages, 2012, doi: $10.5402 / 2012 / 783579$.

[12] B. Kaltenbacher, A. Neubauer, O. Scherzer, Iterative regularisation methods for nolinear ill-posed porblems, de Gruyter, Berlin, New York, 2008.

[13] P. Mahale and M.T. Nair, A Simplified generalized Gauss-Newton method for nonlinear ill-posed problems, Math. Comp. 78 (2009), no. 265, 171-184.

[14] S. Pereverzev and E. Schock, On the adaptive selection of the parameter in regularization of ill-posed problems, SIAM. J. Numer. Anal. 43 (2005), no. 5, 2060-2076.

[15] A.G. Ramm, A.B. Smirnova and A. Favini, Continuous modified Newton'stype method for nonlinear operator equations, Ann. Mat. Pura Appl. 182 (2003), 37-52.

[16] E.V. Semenova, Lavrentiev regularization and balancing principle for solving ill-posed problems with monotone operators, Comput. Methods Appl. Math. (2010), no.4, 444-454.

[17] C.W. Groetsch and A. Neubauer, Convergence of a general projection method for an operator equation of the first kind, Houstan. J. Math. 14 (1988), 201208

[18] A. Krisch, An introduction to the Mathematical Theory of inverse problems, Springer, NewYork, 1996.

[19] S.V. Perverzev and S. Probdorf, On the characterization of self-regularization properties of a fully discrete projection method for Symms integral equation, J. Integral Equat. Appl. 12 (2000), 113-130.

[20] P. Mahale and M.T. Nair, Iterated Lavrentiev regularization for nonlinear ill-posed problems, ANZIAM 51 (2009), 191-217.

[21] U. Tautenhahn, On the method of Lavrentiev regularization for nonlinear illposed problems, Inverse Problems 18 (2002), 191-207.

Addresses: Ioannis K. Argyros: Department of Mathematicsal Sciences, Cameron University Lawton, OK 73505, USA;

Monnanda E. Shobha and Santhosh George: Department of Mathematical and Computational Sciences, National Institute of Technology Karnataka, India-757 025.

E-mail: ioannisa@cameron.edu, shobha.me@gmail.com, sgeorge@nitk.ac.in

Received: 27 February 2013; revised: 29 August 2013 